\title{
Against 'functional gravitational energy': a critical note on functionalism, selective realism, and geometric objects and gravitational energy
}

\author{
Patrick M. Duerr ${ }^{1}$
}

Received: 3 December 2018 / Accepted: 3 December 2019 / Published online: 11 December 2019 (c) The Author(s) 2019

\begin{abstract}
The present paper revisits the debate between realists about gravitational energy in GR (who opine that gravitational energy can be said to meaningfully exist in GR) and anti-realists/eliminativists (who deny this). I re-assess the arguments underpinning Hoefer's seminal eliminativist stance, and those of their realist detractors' responses. A more circumspect reading of the former is proffered that discloses where the so far not fully appreciated, real challenges lie for realism about gravitational energy. I subsequently turn to Lam and Read's recent proposals for such a realism. Their arguments are critically examined. Special attention is devoted to the adequacy of Read's appeals to functionalism, imported from the philosophy of mind.
\end{abstract}

Keywords General relativity · Functionalism · Conservation laws · Energy

\section{Introduction}

This paper scrutinises Read's recent claim that a functionalist strategy can support realism about gravitational energy in General Relativity (GR) - the view that GR possesses local and global gravitational energy-stress in a robust physical sense (at least within a certain class of models). According to Read (2018), such a realism chimes with, and sanctions the use of, gravitational energy common in astrophysical practice.

Read's arguments, I'll argue, aren't convincing - at least not as they stand. In particular, not only is gravitational energy explanatorily dispensable in GR - as Read admits. It is, I submit, a tenuous explanans.

Patrick M. Duerr

patrick.duerr@oriel.ox.ac.uk

1 Oriel College, University of Oxford, Oriel Square, Oxford, Oxfordshire OX1 4EW, UK 
In what follows, I pursue three goals: 1. to critique Read's proposal, 2. to plead for anti-realism about gravitational energy, and 3. to animadvert upon facile uses of functionalism.

The first goal is to push back against Read's realism about gravitational energy: even if one is sympathetic to his overall general argument, one may well question that gravitational energy satisfies its premises.

My second goal is indirect: I'll take up the cudgels for Hoefer's eliminativism about gravitational energy (Hoefer 2000). Hoefer's arguments admit of a different, more circumspect formulation. Thus re-formulated, they evade Read's objections. This also brings to the fore the more serious difficulties that realists about gravitational energy face. I'll argue that Hoefer's eliminativism is ultimately a more satisfactory stance than Read's.

A third goal is to enhance our understanding of functionalism in the philosophy of physics. Section 4 provides a critical discussion of a recent application of functionalism-viz. Read's. This allows us to demarcate more sharply (or, at least, sensitivises us to pitfalls with respect to) what functionalist strategies can and can't achieve- and what suitable contexts for their application might be.

A recurrent theme will be a major, yet somewhat underappreciated question in the extant GR literature ${ }^{1}$ : To what extent is GR special in comparison to, say, electromagnetism or Yang-Mills theories? Advocates of an "egalitarian" view deny this (e.g. Feynman 1995; or Brown 2005). They can rightly point to the-at least, occasional-fertility of such a position. (Think, for instance, of spin-2 derivations of the Einstein Equations, see e.g. Pitts 2016c, or the clarification of the misinterpretation of the cosmological constant as a mass term, Pitts 2019.) Likewise, egalitarians must be given credit for often exposing double standards frequently applied to GR. Contrariwise, advocates of “exceptionalism" affirm GR's privileged status. They accentuate the distinguished explanatory (and, plausibly attendant, ontological) status of GR's spacetime structure (see e.g. Janssen 2009 (whom I take to extend his views on Special Relativity to GR); Nerlich 2007, 2013).

One's realist/anti-realist attitudes towards gravitational energy in GR tend to be fuelled by egalitarian/exceptionalist presuppositions. Usually, they remain implicit. It lies outside the present paper's ambit to adjudicate between GR egalitarianism and GR exceptionalism. Instead, I'll flag where one's verdict on the force of certain (counter-)arguments in the realism/anti-realism debate about gravitational energy hinges on such prior commitments. In this regard, I'll advance the following claim: In terms of realism/antirealism about gravitational energy, Read's functionalism brings nothing new to the table which transcends the realism one may already cherish towards gravitational energy: Only if one is already attracted to realism about gravitational energy (undergirded by egalitarianism about GR), will one find Read's position attractive, too. It doesn't furnish, however, any independent arguments for such a realism.

The paper will proceed as follows. In Sect. 2, I'll introduce the dispute between realists about gravitational energy, such as Read, and antirealists, such as Hoefer. The next section, Sect. 3, hones in on Hoefer's arguments (Sect. 3.1), and Read's

\footnotetext{
1 The most notable and insightful exceptions are found in the oeuvre of Pitts, esp. (2016a, b, 2017, 2019).

Kaiser (1998) reviews the historical dialectics between GR exceptionalism and egalitarianism between 1942 and 1975.
} 
responses to them (Sect. 3.2). Both are assessed in Sect. 3.3. Section 4 is devoted to Read's own realist proposal. I'll first, in Sect. 4.1, reconstruct the logical structure of his argument. Section 4.2 critically evaluates it. Finally, in Sect. 5, I'll outline two contexts not considered by Read: non-tensorial global notions of gravitational energy, and Ashtekar's asymptotics programme.

\section{Setting the stage: Realism about gravitational energy}

In this section, I'll delineate the tenets of realism about gravitational energy, as they appear in the debate between Read (who advocates it) and Hoefer (who discards it).

The debate revolves around the following conundrum. GR's field equations can be derived from varying the Einstein-Hilbert action ${ }^{2}$

$$
\int d^{4} x \sqrt{|g|}\left(\mathcal{L}_{(g)}+\mathcal{L}_{(m)}\right)
$$

The purely gravitational Lagrangian $\mathcal{L}_{(g)}$ is given by the Ricci scalar, $\mathcal{L}_{(g)}=R$, or the so-called, dynamically equivalent (e.g. Hobson et al. 2006, Ch. 19) " $\Gamma \Gamma$ " Lagrangian $\overline{\mathcal{L}}_{(g)}=2 g^{\mu \nu} \Gamma_{\mu[\nu}^{\lambda} \Gamma_{\lambda] \kappa}^{\kappa}$. (It has the advantage of being only first order, in analogy with Lagrangians of other field theories.) $\mathcal{L}_{(m)}$ denotes the matter Lagrangian.

Applying Noether's 1st Theorem (or a suitable generalisation-what Brown and Brading call the "Boundary Theorem") to this total Lagrangian density, $\mathcal{L}_{(m)}+\overline{\mathcal{L}}_{(g)}$, yields a continuity equation (see e.g. Barbashov and Nesterenko 1983 for details):

$$
\partial_{b}\left(\sqrt{|g|}\left(T_{a}^{b}+\vartheta_{a}^{b}\right)\right)=0 .
$$

here $T_{a}^{b}$ denotes the energy-stress tensor associated with ordinary matter fields. $\vartheta_{a}^{b}$ is the canonical energy-momentum associated with the purely gravitational Lagrangian density $\overline{\mathcal{L}}_{(g)}$. It's dubbed the Einstein pseudotensor,

$$
\vartheta_{a}^{b}=\frac{1}{\sqrt{|g|}}\left(-\overline{\mathcal{L}} \delta_{a}^{b}+\frac{\partial \overline{\mathcal{L}}}{\partial\left(\partial_{b} g_{d e}\right)} \partial_{a} g_{d e}\right) .
$$

It transforms tensorially only under affine transformations. Hence, its qualifier "pseudo“. Despite the Einstein pseudotensor's non-tensorial nature, the above continuity holds for all coordinate systems.

Given its exactly analogous construction as canonical energy-momentum in other field theories, the Einstein pseudotensor is naturally construed as local (differential) gravitational energy-stress density. (Below, I'll suppress "density" for the sake of readability.)

In consequence, the above continuity equation is naturally interpreted as local conservation of total energy-stress: Total (gravitational plus matter/non-gravitational)

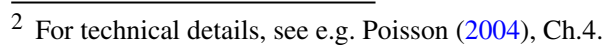


energy-stress, $\mathcal{T}_{a}^{b}:=T_{a}^{b}+\vartheta_{a}^{b}$, has neither sources nor sinks. (A referee has insightfully pointed out that one it might be more correct to say that the (pseudo-)density $\sqrt{|g|} \mathcal{T}_{a}^{b}$ has no sinks/sources: after all, $\sqrt{|g|} \mathcal{T}_{a}^{b}$ —and not $\mathcal{T}_{a}^{b}$ —satisfies the continuity equation. For better or worse, however, I'll stick with the conventional usage in the literature (by Hoefer and Read in particular) that $\mathcal{T}_{a}^{b}$ has no sinks/sources. Nothing in what follows essentially hinges on this.)

Henceforth, I'll refer to this interpretation of the pseudotensor as "realism about

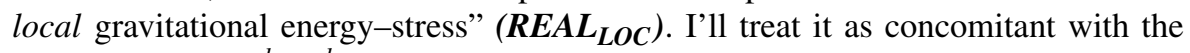
interpretation of $T_{a}^{b}+\vartheta_{a}^{b}$ as local total energy-stress. (For the purposes of this paper, I'll primarily discuss the Einstein pseudotensor as the pseudotensor to which (REAL $L O C)$ is committed. Other choices possible, see below. For the most part, the arguments presented here carry over mutatis mutandis.)

Via an application of Gauß's Theorem, one may now try to convert the continuity equation into a conserved global (integral) quantity over a 4-volume. For the integrals to be well-defined, certain conditions must hold. Preliminarily, I'll subsume them under the label "asymptotic flatness". More details will follow in Sect. 3.1.

The view that this quantity denotes global total energy-stress and is conserved —attendant with the view that the integral over the pseudotensor denotes global gravitational energy-stress - will be referred to as "realism about global gravitational energy-stress and energy-stress conservation" $\left(\boldsymbol{R E A} \boldsymbol{L}_{\boldsymbol{G L O B}}\right)$.

This position is strictly weaker than its local counterpart. Provided one counts a possibly divergent integral as a well-defined, but infinite quantity, one can advocate $\left(R E A L_{G L O B}\right)$ without $\left(R E A L_{L O C}\right)$, but not vice versa. ${ }^{3}$ (Such a situation is familiar from other areas. Think, for instance, of entropy production in thermodynamics. In, say, a Carnot cycle, entropy production $\delta Q_{r e v} / T$, with the reversible heat energy transfer $\delta Q_{r e v}$ and temperature $T$, is defined only up to "thermal gauge transformations", i.e. exact one-forms. Hence, "locally" entropy isn't well-defined. Only "globally" it is, i.e. the integral $\int \delta Q_{\text {rev }} / T$. For a field-theoretical example, think of the selfcurrent of Yang-Mills theories, $j^{A \mu}=-f_{B C}^{A} A_{\lambda}^{B} f^{C \lambda \mu}$. Being explicitly dependent on the connection $A_{\lambda}^{B}$, it's gauge-variant. By contrast, due to the sourceless Yang-Mills equations, $\partial_{\nu} F^{A \mu \nu}=j^{A \mu}$ (with $F^{A \mu \nu}$ denoting the curvature of the connection), the "charge" $Q^{A \mu}=\int d^{4} x \sqrt{|\eta|} j^{A \mu}$ is gauge-invariant.)

Hoefer and Read disagree over whether or not to adopt realism about (local and/or global) gravitational stress-energy. Read affirms both $\left(R E A L_{G L O B}\right)$ and $\left(R E A L_{L O C}\right)$ in certain contexts; Hoefer opposes them without qualification. What are their respective arguments?

\section{Hoefer's eliminativism: read's response}

In this section, I'll first review a straightforward reconstruction of Hoefer's objections to gravitational energy, together with his rejoinder that in GR energy conserva-

\footnotetext{
3 One may regard this, i.e. $\left(R E A L_{G L O B}\right) \& \neg\left(R E A L_{L O C}\right)$, as the orthodox position: Several early authors (e.g. Einstein, 1918, cited in: Gorelik 2002, p. 25; Eddington 1923, p. 137; Pauli 1918, Sect. 61; Weyl 1921, Sect. 31; Schrödinger 1950, p. 100); Misner et al. 1973, Sect. 19-20) reserved realism for global gravitational and total energy-stress.
} 
tion should be abandoned (Sect. 3.1). Subsequently (Sect. 3.2), I'll inspect Read's responses. They are critically evaluated in Sect. 3.3.

The analysis will cast into sharper relief the real problems that (Read's) realism about gravitational energy must address. They'll play a pivotal role in Sect. 4.2.

\subsection{Hoefer's eliminativism}

In favour of eliminativism about gravitational energy and energy conservation, $\neg\left(R E A L_{G L O B}\right) \& \neg\left(R E A L_{L O C}\right)$, Hoefer mounts three arguments: $(H 1)$ coordinatedependence, (H2) ambiguity, and (H3) inapplicability of the conditions that the definability of gravitational energy presupposes.

Hoefer's first point, $(\mathrm{Hl})$, is that realism about energy-stress conservation "goes against the most important and philosophically progressive approach to spacetime physics: that of downplaying coordinate-dependent notions and effects, and stressing the intrinsic, covariant and coordinate-independent as what is important" (pp. 194).

According to Hoefer, the pseudotensor featuring in $\left(R E A L_{L O C}\right)$ doesn't comply with this precept: "(I)ts non-tensorial nature means that there is no well-defined intrinsic 'amount of stuff' present at any given point" (ibid.). Neither does $\left(R E A L_{G L O B}\right)$ comply - presumably because asymptotic flatness, as Hoefer presents it, is formulated via the following coordinate conditions:

(a) For $r:=\sqrt{x^{2}+y^{2}+z^{2}} \rightarrow \infty$, the coordinate system must be asymptotically Lorentzian, i.e. $g_{\mu \nu} \rightarrow \eta_{\mu \nu}=\operatorname{diag}(1,-1,-1,-1)$. In the interior, it can vary arbitrarily.

(b) The metric must decay sufficiently rapidly: $g_{\mu \nu} \rightarrow \eta_{\mu \nu}+\mathcal{O}(1 / r), \partial g_{\mu \nu} \rightarrow \mathcal{O}$ $\left(1 / r^{2}\right), \partial^{2} g_{\mu \nu} \rightarrow \mathcal{O}\left(1 / r^{3}\right)$.

Hoefer's second objection, (H2), (ibid.) attacks the pseudotensor's ambiguity: It's not uniquely defined. Some elaboration is in order of what Hoefer may have had in mind. The pseudotensors are defined only up to a transformation of the form $\vartheta_{a}^{b} \rightarrow \vartheta_{a}^{b}+\partial_{c} \Xi_{a}^{[b c]}$. Here, $\Xi_{a}^{[b c]}$ is a so-called superpotential, anti-symmetric in its upper indices (see Trautman 1965, for details). As a result it's vastly underdetermined, thereby impeding $\left(R E A L_{L O C}\right)$.

Hoefer's third argument, $(H 3)$, targets $\left(R E A L_{G L O B}\right)$. His thought seems to be that $\left(R E A L_{G L O B}\right)$ hinges on realism about the conditions under which it's well-defined, i.e. asymptotic flatness. Hoefer correctly observes that our actual world isn't asymptotically flat. Realism about asymptotic flatness thus is mistaken. This, according to Hoefer, undercuts $\left(R E A L_{G L O B}\right)$.

Hoefer's point straightforwardly carries over to $\left(R E A L_{L O C}\right)$. In flat spacetimes, pseudotensors are to some extent extricated from their unsettling non-tensorial transformation behaviour: In them, Poincaré transformations-i.e. at least a subgroup of linear transformations - are distinguished as relating physically equivalent frames. Alas, Hoefer might interject: Our universe isn't flat-not even asymptotically. An advocate of $\left(R E A L_{L O C}\right)$ thus has to stomach non-tensoriality.

Based on his diagnosis of coordinate-dependence, ambiguity and anti-realism about the formal prerequisites for defining global gravitational energy, Hoefer champions 
antirealism/eliminativism about gravitational energy: we should relinquish the notion. Instead, we should just accept that in GR, energy conservation no longer holds.

How does Read respond to Hoefer's arguments?

\subsection{Read's response}

While for Hoefer the above reasons suggest that one abandon $\left(R E A L_{G L O B}\right)$ and $\left(R E A L_{L O C}\right)$, Read wants to resist this conclusion. He parries by $(R I)$ rejecting Hoefer's ban on coordinate-based language, $(R 2)$ by assuming that the non-uniqueness can be overcome (or, at least, isn't problematic), $(R 3)$ by defending the use of idealisations, and $(R 4)$ by baulking at the revisionary nature of Hoefer's eliminativism.

First, Read takes Hoefer to outlaw the usage of coordinate-dependent notions ( $\mathrm{Hl}$ ). Read rightly repudiates this as unwarranted $(R l)$. The mere usage of coordinates is unproblematic ${ }^{4}$ : "[...] (P)resentations of spacetime theories need not proceed in a coordinate-independent manner; rather, spacetime theories may be defined in terms of equations written in a coordinate basis and their transformation properties (this is what Brown [...] and Wallace [...] refer to as the 'Kleinian conception of geometry'), and explanations may be given by appeal to those laws, written in a coordinate basis." On this Kleinian conception, one characterises geometry via the class of privileged coordinate systems (see Wallace 2016 for details). In these, the dynamical equations preserve a particular (e.g. simplest) form. Such coordinate-based characterisations are as coordinate-independent as those not based on coordinates, i.e. drawing on intrinsically geometric notions. Prima facie, Read thus effectively wards off Hoefer's first complaint.

It might even appear that $(H 1)$ was unfounded for $\left(R E A L_{G L O B}\right)$ from the outset: While it's popular and expedient to define asymptotic flatness in a coordinate-based manner (e.g. Jaramillo and Gourgoulhon 2010 for a more detailed presentation), this isn't necessary. Via conformal techniques, it's indeed possible to characterise asymptotic flatness in purely geometric, coordinate-free terms (e.g. Geroch 1972, Ch. 35-38; Wald 1984, Ch. 11; Ludvigsen 1999, Ch. 12)—as Hoefer demands.

To Hoefer's complaint of the ambiguity of pseudotensors (H2), Read responds as follows $(R 2)$ : "There are many distinct but non-equivalent choices for this pseudotensor, based on one's choice of superpotential. Hence [...] we are implicitly supposing that a choice has been made from the family of possible candidates" (p. 11). (Below, I'll also consider a different response that Read may be read as endorsing.)

In his third response, $(R 3)$, Read rebuts Hoefer's attack on asymptotic flatness as an assumption not applicable to our universe (H3). Read acknowledges: It is "[...] undeniable [...] that the entire universe is not asymptotically Minkowski" (pp. 16). Yet, according to Read, asymptotic flatness is a good idealisation for certain approximately isolated subsystems. ${ }^{5}$

\footnotetext{
${ }^{4}$ Geometric/coordinate-free formulations are even ill-suited for applications of GR's initial value problem (see, e.g., Isenberg 2014).

5 Characterising the domain of applicability of asymptotic flatness as "systems within the world [...] considered in isolation" (Read 2018, p. 17) verges on being tautological (cf. Curiel 2000, pp. 17): One
} 
Read rightly underscores that "every theory of physics is an idealisation and does not 'apply to the actual world' in this strong sense" (p. 17). He takes Hoefer to reject asymptotic flatness as an ultimately inaccurate assumption. That, however, Read argues, demands too much of successful hypotheses for them to earn realist commitments: Ultimate exactness is never attainable. Rather, Read suggests that this doesn't curtail the utility of asymptotic flatness as an idealisation.

Read's final response, $(R 4)$, is to avoid Hoefer's eliminativism due to its "potentially undesirable consequences". On the one hand $(R 4 a)$, "such a claim would also commit one to the statement that there exists no genuine stress-energy conservation law in [Special Relativity, SR] - a theory in which the conservation of total stress-energy typically is taken to be uncontroversial" (p. 18). On the other hand $(R 4 b)$, "the advocate of the Hoefer-type view is apparently committed to the denial of the claim that gravitational waves and other forms of purely gravitational radiation are energetic". Read avers that this is gratuitously revisionary.

Do Read's responses - the legitimacy of coordinate-based language, the implicit supposition that the non-uniqueness can be overcome, the legitimacy of asymptotic flatness as an approximation, and the rebarbative ramifications of Hoefer's eliminativism-effectively rebut Hoefer's worries? In the next paragraph, I'll assess Read's answers, arguing that they don't.

\subsection{Hoefer reloaded}

I'll now critically examine Read's counters to Hoefer, $(R I)-(R 4)$. Each, I submit, misses the more subtle points of Hoefer's critique: $(R l)$ conflates the mere usage of coordinates with a vicious coordinate-dependence; $(R 2)$ merely voices a hope, not an argument; $(R 3)$ ignores the distinction between approximations and idealisations; $(R 4)$ is in part, both exegetically and systematically unwarranted, and in part an appeal to majority consensus.

Let's begin with $(R 1)$, Read's rehabilitation of coordinate-based descriptions à la Klein. I deem it a red-herring: It's an infelicity in Hoefer's presentation of his argument which invites the misunderstanding that Hoefer wishes to ban coordinate-based language per se. A more disconcerting real issue lurks behind his worry, though: Pseudotensors, which figure in $\left(R E A L_{L O C}\right)$, are artefacts of conventions; something akin besets the integral over them.

Read is certainly right in that neither coordinate-relativity nor non-tensoriality need prevent us from ascribing an object physical significance. The Levi-Civita connection coefficients, $\Gamma_{\mu \nu}^{\lambda}=\frac{1}{2} g^{\lambda \sigma}\left(\partial_{\nu} g_{\sigma \mu}+\partial_{\mu} g_{\sigma \nu}-\partial_{\sigma} g_{\mu \nu}\right)$, attest to that: Geometrically,

\section{Footnote 5 continued}

defines a general-relativistic system as (materially and gravitationally) isolated because its total energy content is conserved; otherwise, one would regard it as (at least) gravitationally interacting.

To avoid this vacuity, I take Read to make the more specific claim that certain subsystems of the universe that don't interact non-gravitationally are approximately asymptotically flat.

To jump ahead a little: The preceding claim can't be universally true-as witnessed by textbook FLRW cosmologies: Their matter sector is modelled by cosmic dust, i.e. a homogeneous, isotropic fluid with negligible non-gravitational interactions, see e.g. Hobson et al. (2006, Ch. 14). One therefore ought to understand Read's claim as this: There exists a physically relevant, and empirically well-corroborated class of only gravitationally interacting systems that are asymptotically flat. We'll return to this in Sect. 4. 
they connect the fibers of the tangent bundle over different points of the base manifold; physically, they encode inertial structure.

Yet, pseudotensors are plagued by "vicious coordinate-dependence" (Pitts 2009, p. 16) ${ }^{6}$ : They pick out preferred coordinates in the above Kleinian sense that don't align with the spacetime symmetries. Equations involving pseudotensors preserve their invariance only under affine coordinate transformations. But what distinguishes them in non-flat spacetimes? (Recall: In generic spacetimes affine coordinate transformations aren't preferred in the Kleinian sense.) Conversely, how to make sense of the fact that pseudotensors don't respect spacetime symmetries? That is, how to understand the fact that their invariance isn't preserved under spacetime symmetry transformations - in contradistinction to what one expects of matter fields (cf. Pooley 2013)?

These oddities are highlighted by the fact that pseudotensorial 4-fluxes of gravitational energy-momentum, $\vartheta_{\mu}^{\nu} \xi^{\mu}$ (along the direction of $\xi$ ) don't transform like 4-vectors under purely spatial, or under purely temporal transformations. But both amount to a merely conventional re-labelling of points in space, and continuous change in the rate and setting of a coordinate clock (Horský and Novotný 1969, p. 431), respectively. Neither should impact physical quantities — such as energy-momentum fluxes.

By stressing merely their non-tensoriality, Read downplays the viciousness of pseudotensors: They aren't merely (and, as the Kleinian hastens to add: benignly) "frame-relative"; they are viciously frame-dependent. This has its exact counterpart in the frame-dependence of non-invariant quantities in SR: The latter don't represent objective features of the world; reifying them leads to the notorious "paradoxes" of SR (see Maudlin 2011, Ch. 2, 2012, Ch. 4 for lucid explications).

It's instructive to re-phrase the problem: Pseudotensors don't form geometric objects in the sense of e.g. Trautman $\left(1965\right.$, Ch. 4.13, 1962). ${ }^{7}$ A geometric object $y$ on an $N$-dimensional manifold $\mathcal{M}$ is a correspondence $y: p,\left\{x^{\mu}\right\} \rightarrow y_{1}, \ldots, y_{N} \in \mathbb{R}^{N}$ which associates with every point $p \in \mathcal{M}$ and every local coordinate system $\left\{x^{\mu}\right\}$ around $p$ an $N$-tuple $y:=y_{1}, \ldots, y_{N}$ of real numbers (the object's components), together with a definite transformation rule that relates the components relative to the original coordinate system, and the components $y^{\prime}:=y_{1}^{\prime}, \ldots, y_{N}^{\prime}$ relative to a different coordinate system $\left\{x^{\prime \mu}\right\}$ around $p$. The transformation rule only involves the object's components $y, y^{\prime}$ relative to the coordinate systems $\left\{x^{\mu}\right\}$ and $\left\{x^{\prime \mu}\right\}$, and their Jacobi matrix $\partial x / \partial x^{\prime}$.

Requiring that the transformation rule depend only on the the components and the Jacobi matrix and the Jacobi matrix's gradient (i.e. $y, y^{\prime}, \partial x^{\prime} / \partial x, \partial^{2} x^{\prime} / \partial x^{2}$ ) is necessary for the mutual consistency of legitimate coordinate systems in the following sense: Whenever we can use different coordinate systems, the order in which we switch from one to the other them doesn't matter. This can be stated more precisely. Let the transformation rule $T\left[y, y^{\prime}, \partial y / \partial x, \partial x / \partial x^{\prime}\right]$ ) for the coordinate transformation $\left\{x^{\mu}\right\} \rightarrow\left\{x^{\prime \mu}\right\}$ depend on, say, $\partial y / \partial x$. Consider now $\left\{x^{\prime \prime}\right\}$ as a third coordinate

\footnotetext{
6 Pitts propounds a method to construct geometric, infinite-component objects out of pseudotensors (see below). Those overcome the vicious coordinate dependence-at least formally.

7 The notion of geometric object objects was standard in differential geometry from the $1930 \mathrm{~s}$ onward. (It shouldn't be conflated with Anderson's related programme of analysing substantive general covariance in terms of absolute objects, cf. e.g. Pitts 2006).
} 
system. Suppose that relative to it, $\left.T\left[y^{\prime}, y^{\prime \prime}, \partial y^{\prime} / \partial x^{\prime}, \partial x^{\prime} / \partial x^{\prime \prime}\right]\right)$ also holds. In general, it won 't follow, however, that $T\left[y, y^{\prime \prime}, \partial y / \partial x, \partial x / \partial x^{\prime \prime}\right]$ ) is satisfied (for details, see Kucharzewski and Kuczma 1964).

In short: The components of geometric objects in arbitrary coordinates are uniquely determined by their components in one coordinate system and the transformations between the coordinates.

By way of example, note that connection coefficients $\Gamma_{\kappa \lambda}^{\mu}$ form a geometric object. Under coordinate changes, they transform as

$$
\Gamma_{\kappa \lambda}^{\mu} \rightarrow \Gamma_{\kappa \lambda}^{\prime \mu}=\frac{\partial x^{\prime \mu}}{\partial x^{\prime}} \frac{\partial x^{\sigma}}{\partial x^{\prime \kappa}} \frac{\partial x^{\tau}}{\partial x^{\prime \lambda}} \Gamma_{\sigma \tau}^{\rangle}+\frac{\partial^{2} x^{\sigma}}{\partial x^{\prime \kappa} \partial x^{\prime \lambda}} \frac{\partial x^{\prime \mu}}{\partial x^{\sigma}} .
$$

By contrast, consider the vector field $v^{\mu}$. Then, the quantity $\frac{\partial v^{\mu}}{\partial x^{v}}$ doesn't form a geometric object: Under coordinate changes, it transforms as

$$
\frac{\partial v^{\mu}}{\partial x^{\nu}} \rightarrow \frac{\partial v^{\prime \mu}}{\partial x^{\prime \nu}}=\frac{\partial x^{\prime \mu}}{\partial x^{\kappa}} \frac{\partial x^{\lambda}}{\partial x^{\prime \nu}} \frac{\partial v^{\kappa}}{\partial x^{\lambda}}+\frac{\partial x^{\lambda}}{\partial x^{\prime \nu}} \frac{\partial x^{\prime \mu}}{\partial x^{\kappa} \partial x^{\lambda}} v^{\kappa}
$$

That is: The transformation rule exhibits the prohibited dependence on $v^{\kappa}$ (rather than $\frac{\partial v^{\kappa}}{\partial x^{\lambda}}$ ). One can indeed straightforwardly verify that in virtue of this dependence, successively applying the preceding transformation law to two coordinate transformations, $\left\{x^{\mu}\right\} \rightarrow\left\{x^{\prime \mu}\right\}$ and subsequent $\left\{x^{\prime \mu}\right\} \rightarrow\left\{x^{\prime \prime \mu}\right\}$ yields a transformation law, different from the one for the coordinate transformation $\left\{x^{\mu}\right\} \rightarrow\left\{x^{\prime \prime \mu}\right\}$.

In the same sense, being non-geometric objects, pseudotensors are viciously coordinate-dependent: The transformation rules of pseudotensors exhibit a dependence on the coordinates employed. ${ }^{8}$ The consistency condition is violated.

Geometric objects, however, constitute the standard framework within which physical objects of contemporary field theories are couched (see Nijenhuis 1952; Schouten 1954; Anderson 1967, 1971; Torretti 1996, Ch. 4.3). ${ }^{9}$ They ensure that the intrinsic properties of physical entities and all relations between them are preserved under general coordinate transformations-the mere re-labelling of the manifold points: "Thus the components of a geometric object form a natural kind mathematically: they constitute faces of one and the same entity by virtue of being interrelated by a coordinate transformation law" (Pitts 2009, p. 610). (Note that this is compatible with the existence of special coordinates, in which the physical laws take a particularly simple form.) By contradistinction, the properties and relations of entities represented by non-geometric objects are, as it were, sensitive to the labels attached to spacetime points. But such labels are usually deemed merely conventional. (Equivalently: Non-geometric objects presuppose more structure-information encoded directly in coordinates of the manifold points - than a manifold, standardly construed, contains.) Due to their non-geometric nature, the physical significance of pseudotensors, and hence the tenability of $\left(R E A L_{L O C}\right)$, thus becomes questionable.

\footnotetext{
8 NB: Generically each pseudotensor has a different (viciously coordinate-dependent) transformation rule.

9 It's worthwhile mentioning that Friedman (1983), in contrast to the cited authors, restricts geometric objects to either tensors or connections. Thereby he-unduly-neglects, for instance, tensor densitities of arbitrary weight or projective connexions (cf. Pitts 2006, 2012; Schouten 1954, p. 301).
} 
To be sure, Read could stand by his guns: He might withdraw his allegiance to the geometric object programme. ${ }^{10}$ Suppose that a pseudotensor $\theta_{a}^{b}\left[\tau, \Sigma_{\tau}\right]$ is only meaningful relative to a given coordinate system. Let the latter represent a $(3+1)$ decomposition ("frame"), $\tau, \Sigma_{\tau}$. Relative to a different frame, $\tau^{\prime}, \Sigma_{\tau}^{\prime}$ one obtains a distinct object, $\theta_{a}^{\prime b}\left[\tau^{\prime}, \Sigma_{\tau}^{\prime}\right]:=\theta_{a}^{b}\left[\tau^{\prime}, \Sigma_{\tau}^{\prime}\right]$. Vicious coordinate dependence of $\theta_{a}^{b}$ now has lost its sting: $\theta_{a}^{b}$ and $\theta_{a}^{\prime b}$ represent distinct entities.

What impedes the interpretation of such frame-relative objects is that no (3 +1 )-decomposition is distinguished over any other. To preserve this "frameegalitarianism", one has two options. The first one is to renounce realism about those $\theta_{a}^{b}\left[\tau, \Sigma_{\tau}\right] \mathrm{s}$ for all possible frames. This is tantamount to anti-realism towards pseudotensors. The second option is to extend one's realist attitude to every $\theta_{a}^{b}\left[\tau, \Sigma_{\tau}\right]$ for all frames. The idea is to lump the totality of all $Q\left[\tau, \Sigma_{\tau}\right]$ s for all possible frames, $\left\{\tau, \Sigma_{\tau}\right\}$, into one formal object-symbolically:

$$
\Theta:=\left\{\theta_{a}^{b}\left[\tau, \Sigma_{\tau}\right]: \forall \tau, \Sigma_{\tau}\right\}
$$

(Think of each $\theta_{a}^{b}\left[\tau, \Sigma_{\tau}\right]$ as one of the uncountably infinitely many components of $\Theta$.) A realist about $\Theta$ doesn't privilege any of its components. Thereby, she respects frame-egalitarianism. ( $\Theta$ is even a geometric object in a slightly relaxed sense. $\left.{ }^{11}\right)$ Pitts (2009) has indeed made this astute proposal.

Here, we needn't arbitrate between the anti-realist first, or the realist second option. It's clear, however, that at this stage $\left(R E A L_{L O C}\right)$ is staked on the plausibility of Pitts' proposal. Whether the latter is persuasive remains to be seen (cf. Curiel 2018, fn 27; Duerr 2018a, Sect. 3.3 for a critique). Read, at any rate, stays silent on the matter. (Plausibly, a defence of realism about Pitts' proposal deploys a double strategy akin to Read's: 1. to appeal to scientific utility to licence realist stance towards it, and 2. to appeal to similarities with pre-relativistic notions of gravitational energy in order to identify Pitts' object as their genuine, general-relativistic analogue. Read's crucial - to-date unaccomplished - task would then be to flesh all of this out in detail.)

In the same vein, Read's Kleinian vindication of coordinate-use doesn't allay a related worry for $\left(R E A L_{G L O B}\right)$. Different coordinate choices can give rise to different (or even ill-defined) distributions of global (gravitational) energy-stress (see Xulu

\footnotetext{
10 Contra Read's (2018, Sect. 3.1) remark, absent any explicit discussion in his work (to my knowledge), it's hard to say-and possibly a rewarding reconstructive task, integrating his views on coordinates (see e.g. Norton 1989, 2002) and interpretation of GR (see e.g. Lehmkuhl 2014)—whether Einstein himself would have had qualms about non-geometric objects. While he repeatedly objected to the requirement that all meaningful objects be tensorial, that view is, as we saw, compatible with an insistence on geometric objects. Indeed, Torretti (1996, p. 316, fn 1) views Einstein's insistence on a "definite transformation rule" as essentially an endorsement the geometric object framework. (This is compatible with the passage, cited by Read (fn 22), in which Einstein defends his pseudotensor against his colleagues' complaint. Therein, Einstein (1918, p. 449) critiques their view that "all physically significant quantities can be understood as scalars and tensor components" (my translation). It's not clear, however, that Einstein fully understood the non-geometric nature of his pseudotensor (avant la lettre). Yet, later on (p. 452) in the cited text, Einstein seems to concede some unease about his pseudotensor: "[...] we thus come to ascribe more reality to the integral than to its differentials" (my translation). I thank James Read for pressing me on this.).

11 Usually (e.g. Trautman 1965, p. 85 or Anderson 1967, p. 15) one considers only geometric objects with finite components.
} 
2003 , for a survey of explicit calculations). To maintain realism about pseudotensorbased integral quantities, one must cope with the ambiguity resulting from such coordinate-dependence.

There are three options. The first is to remove the ambiguity by privileging certain coordinate systems (e.g. quasi-Cartesian ones). This seems to contravene frameegalitarianism. (On the other hand, Read might argue—as does e.g. Pitts (2009)—that our world, to a good approximation, does privilege quasi-Cartesian coordinates anyway. But first, one may worry whether such an appeal to approximate symmetries is sufficiently robust: How good need the approximation be for it to legitimately privilege quasi-Cartesian coordinates? Secondly, as we'll see below (Sect. 4.3.2), quasi-Cartesian coordinates aren't privileged, when applied to the universe as a whole-nor generic subsystems. If, however, one restricts oneself to not too large spacetime regions that can be approximated as roughly Minkowskian, quasi-Cartesian coordinates are indeed privileged for those subsystem. But such a quasi-Minkowksian regime is contingent, and fairly arbitrarily stipulated: What then justifies Read in distinguishing it for characterising gravitational energy and/or energy conservation?)

The other two options are in line with it. One is to adopt anti-realism about such integral quantities. This defeats Read's realist ambitions. He should therefore pursue the third option - the integral/global version of Pitts' proposal: All integral quantities are real. That is, he should adopt realism about infinite-component objects of the (quasi-symbolic) type

$$
\int \Theta:=\left\{\int d V \theta_{a}^{b}\left[\tau, \Sigma_{\tau}\right]: \forall \tau, \Sigma_{\tau}\right\} .
$$

As before in the case of $\left(R E A L_{L O C}\right)$, the plausibility of Read's version of $\left(R E A L_{G L O B}\right)$ hinges on the plausibility of realism towards such objects. Again, Read's position doesn't add a new argument for $\left(R E A L_{G L O B}\right)$. Rather, it crucially relies on a prior commitment to a realism about the integral Pitts-object-for which no argument is given.

In summary: A conservative framework for classical field theories is Anderson's geometric objects programme. Within it, a physical interpretation of pseudotensors, as envisaged by $\left(R E A L_{L O C}\right)$, is doubtful: They aren't geometric objects. $\left(R E A L_{G L O B}\right)$ fares no better: Pseudotensor-based global notions of gravitational energy are coordinate-dependent artefacts of conventions. Read has two options: either to reject (or revise) the geometric object framework, or to extend his realism to Pitts' object. For either choice, we are owed an argument. These problems hold irrespective of one's predilection for a Kleinian or a coordinate-free approach to geometry. Read's response $(R I)$ cuts no ice against them.

Let's continue with $(R 2)$, Read's second response, regarding the pseudotensor's ambiguity (H2): He simply assumes that one can learn to live with the plurality, or that uniqueness can be restored in a principled manner.

The ambiguity of pseudotensors bodes ill for $\left(R E A L_{G L O B}\right)$ : Different pseudotensors can also yield different global energy distrutions (see again Xulu 2003, also for further references). 
One response is, of course, to accept the ambiguity about gravitational energy-stress. But such pluralism has a drastic conclusion: Via the First Law, it threatens to subvert the uniqueness of thermodynamic states more generally. Read shies away from this (pers.comm.).

Read's hopes should therefore be set on a way of coping with the non-uniqueness. But he remains silent on how to achieve this. Why believe Read's "implicit supposition" (R2)?

Two possible reasons spring to mind: One is that perhaps uniqueness can be restored; the other is to bite the bullet: Perhaps the non-uniqueness is a feature, not a bug.

It's certainly conceivable that the list of viable pseudotensors can be further whittled down. For instance, vis-à-vis its anomalous factor, it's plausible to exclude the Møller pseudotensor (Katz 1985). More general arguments for a unique expression are collated in works by Katz (2005), Katz et al. (1997) and Petrov (2008). (Note that these authors use a background metric. Vis-à-vis such auxiliary structure one may already ponder: Does its introduction compromise the result?) While an enticing project, a comprehensive analysis of such an agenda is pending.

Another possibility for coping with the non-uniqueness is "to try to find meaning in it" (Pitts). In this spirit, Pitts (2017), following Nester (2004) and collaborators, suggests that the pseudotensors' ambiguity is a blessing in disguise: Their differences correspond to different free energies and the like under different boundary conditions. It remains to be seen whether this proposal is convincing (cf. Duerr 2018a, p. 11). At present, it too is an enticing project, not a clear-cut argument in Read's favour.

In short: As it stands, Read's response (R2) falls short of being an argument. At present, whether uniqueness for gravitational energy can be restored is an open question. Likewise, whether non-uniqueness is an advantage, remains controversial.

Read's third response (R3) takes Hoefer to reject asymptotic flatness for not applying to our universe. Read seeks to legitimise its use as an approximation. This way of portraying Hoefer's criticism, however-as a demand for excessive rigour-glosses over a deeper concern: To what extent may we assume that the universe possesses the relevant structures that gravitational energy presupposes?

We can render the question's import more transparent by dint of Norton's distinction between approximations and idealisations (Norton 2012). The former denotes an inexact description of the target system. The approximation's referent coincides with it. An idealisation, by contrast, is an (inexact) description of a surrogate system that mimics the target system in relevant regards. An idealisation's referent is thus distinct from the target system.

Given a supremely successful model, an inference to the best explanation (IBE) entails different realist stances towards it—depending on whether one classifies it as an approximation or an idealisation (cf. ibid, Sect. 2.4; Torretti 1990, Ch. 3.6). In the first case, an IBE licences realism about the model totaliter: The target system can be assumed to actually possess, at least roughly, the properties of the model. By contradistinction, an IBE about an idealisation licences only a "selective realism": We may only assume that the target object shares some structural features with the model-those responsible and indispensable for the model's explanatory success, its "working posits" (see e.g. Vickers 2016, 2017 for details). Only they-not the model tout court-merit realism. 
Norton's distinction affords a refined reading of Hoefer's objection to asymptotic flatness: Rather than an intolerably imprecise approximation, asymptotic flatness is an idealisation of our actual world. Even when successful, an IBE about asymptotically flat models consequently doesn't warrant an unqualified realism: Only their working posits merit realism. Hoefer's criticism $(H 3)$ is thus best construed as the view that asymptotic flatness is an idle posit of an idealisation. By contrast, Read's response (R3) is more sanguine: Asymptotic flatness is either an approximation, or a working posit of an idealisation. Hence, it literally (albeit only in approximation) depicts real structures in the world. Neither Hoefer nor Read proffers arguments for their respective verdicts, thus construed. I'll arbitrate between them in Sect. 4.2.

To summarise: Hoefer's objection to asymptotic flatness is best interpreted as the view that the explanatory successes of relativistic astrophysics and cosmology don't justify the belief in approximate asymptotic flatness. Read champions this belief. Neither backs up his stance by arguments.

With his fourth response, $(R 4)$, Read goes on the offensive. He points to two unappealing alleged consequences of Hoefer's position. Neither argument strikes me as cogent.

Read's first claim, $(R 4 a)$, is that Hoefer's eliminativism implies also a failure of energy-conservation in Special Relativity (SR) - provided one demands that an acceptable conservation principle hold in every frame. I agree with Read about this conditional claim. But I find no textual evidence that Hoefer endorses the antecedent condition. ${ }^{12}$ But even if he did: His eliminativism isn't inherently tied to the (implausible) doctrine that conservation principle must hold in all frames. ${ }^{13}$ In fact, for the same reasons why fictitious forces in Classical Mechanics (e.g. the Coriolis force) are artefacts of descriptions in ill-adapted, generic coordinate systems that needn't disturb us (see e.g. Maudlin 2012, pp. 23, fn. 7), we needn't be worried by a conservation principle formulated in special coordinate systems.

If we thus drop the doctrine of equality of all frames, SR's conservation law remains untouched-as Read (2018, Sect. 2.4) admits: Owing to the existence of a timelike Killing field in Minkowski spacetime, a bona fide, tensorial local and global conservation law is straightforward (e.g. Straumann 2013, Ch. 3.4). The coordinates adapted to these symmetries are the familiar (globally defined) Lorentz coordinates. In them the matter energy-stress tensor satisfies an ordinary continuity equation, with its standard interpretation.

Read's second claim, $(R 4 b)$, is that on Hoefer's view, the standard interpretation of binary systems must be jettisoned: In this account, gravitational energy evidently is a central explanans (e.g. Hobson et al. 2006, Ch. 18). An eliminativist about gravitational energy, however, abjures it. Read's lesson: So much the worse for Hoefer's

\footnotetext{
12 The two passages that might suggest the contrary are the following. In the first one, (p. 193), Hoefer says about the validity of the pseudotensor-based continuity equation in all frames that "[...] (it) can be taken as generally covariant." This suggests that he equates general covariance with validity in arbitrary coordinate-systems. But in light of the Kretschmann argument (e.g. Norton 1985, Sect. 5, whom Hoefer cites!), this sense of general covariance is merely formal: Any theory can be made to conform to it.

The second passage is along similar lines. Hoefer (p. 195) approvingly quotes Stephani: The latter laments the non-tensoriality of pseudotensorial gravitational energy as a violation of general covariance.

13 This doctrine is arguably a relic of Einstein's (erroneous) initial understanding of general covariance (see again Norton 1985 for details).
} 
eliminativism. But Read's argument is an argumentum ad verecundiam: It merely cites orthodoxy in the community. What are the cogent, systematic reasons to subscribe to it? I'll return to this in Sect. 4.

In short, neither claim comprising $(R 4)$ is persuasive: $(R 4 a)$ implausibly imputes to Hoefer an implausible doctrine; $(R 4 b)$ is an appeal to majority.

The insights gained here will pave the ground for our discussion of Read's own position-the topic of our next section.

\section{Functional Gravitational Energy and its discontent}

Here, I'll first (Sect. 4.1) lay out Read's functionalist approach to gravitational energy. Its logical structure will be made explicit. Subsequently, (Sect. 4.2) I'll critically examine three of its crucial premises. I reject them for multiple reasons. Notwithstanding my sympathies to his overall functional approach, and to the Dennettian ontological framework, I conclude that Read's realism should be dismissed.

\subsection{Functional Gravitational Energy}

Here, I'll expound Read's realism about gravitational energy-stress (Read 2018, Sects. 3.3.2, 3.3.3), and the logical structure of his argument for it. Read proposes to embrace the background relativity of gravitational stress-energy (in the sense of Sect. 3.3). As this background-relative notion is both useful, and satisfies the functional role of gravitational, according to Read, we should be realists about it.

By "background" Read (and Lam, see below) mean (asymptotic) symmetries, encapsulated in asymptotic Killing fields, and suitable fall-off conditions, both implemented via asymptotic flatness. Lam and Read suggest that one should regard local and global gravitational and total energy as quantities well-defined relative to this background.

Let's unravel his reasoning in more detail. Read picks up an earlier intimation by Lam (2011): On the one hand, "[...] within [GR] all meaningful notions of (gravitational and nongravitational) energy-momentum [...] require the introduction of some background structures" (p. 1023); on the other hand, if these structures are present, genuine gravitational and non-gravitational energy exists: "they make only sense in particular (but very useful) settings" (ibid.).

Read's realism, $\left(R E A L_{L O C}\right)$ \& $\left(R E A L_{G L O B}\right)$, can now be cashed out as positive, principled answers to the following two questions (p. 19):

(a) Does the pseudotensor $\vartheta_{a}^{b}$ in $\left(R E A L_{L O C}\right)$ and its associated integral ("charge") in $\left(R E A L_{G L O B}\right)$ represent anything real? Are these formal terms grounded in physical (but not necessarily fundamental) quantities?

(b) Suppose a positive answer to (a). Are we then licenced to identify the quantities that $\vartheta_{a}^{b}$ and its associated charge represent as gravitational energy-stress? "(I)s it correct to call the quantity appearing in [the continuity equation of (REAL $\left.L_{L O C}\right)$ and its integral form in $\left.\left(R E A L_{G L O B}\right)\right][\ldots]$ 'gravitational stress-energy"'? 
The questions in (a) require a reality criterion. Echoing Lam, Read appeals to the explanatory and predictive utility of the gravitational pseudotensor and its associated charge: "[...] (they) are only well defined in a certain subset of [dynamically possible models, DPMs] of GR"; (n)evertheless, in such instances it is extremely useful to make use of this term, within that subclass of DPMs. Hence, at a practical level, it is legitimate to call such a quantity gravitational stress-energy."

This is an instance of the following principle for realist commitment towards a theoretical, higher-level concept $Q$ (cf. Dennett 1991a; Ladyman and Ross 2007, esp. Ch. 4)—what Wallace (2012, Ch. 2) dubs "Dennett's Criterion":

(DC) Whenever $Q$ is definable and explanatorily or predictively useful, it captures a real structure ("real pattern") in the world.

Real patterns are higher-level structures: They are formulated in non-fundamental terms. (Think of molecules and their shapes as treated in chemistry. A satisfactory fundamental account isn't available at present (see Hettema 2012 for the chemical case). Of course, this doesn't imply that real patterns are "strongly autonomous" (Fodor), i.e. unrelated to the most fundamental level.)

To complete his affirmative answer to (a), Read needs to assume that the quantities conventionally labelled "(formal) gravitational energy", $\operatorname{grav}_{f}{ }^{14}$ indeed satisfy $(D C)$ :

$(D C)\left[g r a v E_{f}\right] \quad$ For certain DPMs, gravE $E_{f}$ is definable and explanatorily/predictively useful.

It now follows from $(D C)$ that $g r a v E_{f}$ captures a real pattern in the world ("is real"):

$$
(D C) \&(D C)\left[\operatorname{gravE}_{f}\right] \rightarrow \operatorname{gravE}_{f} \text { is real. }
$$

Having established the reality of formal gravitational energy, Read's next step is to affirm (b): The real pattern gravE $E_{f}$ captures should be identified as genuine gravitational energy-stress; it represents gravitational energy-stress also in a substantive, physical sense.

Read's rationale encompasses three elements: a general functionalist principle for characterising quantities, a particular functional profile for genuine gravitational energy-stress, and the premise that $g r a v E_{f}$ exhibits this profile.

Read deploys what he terms a "functionalist" (p. 20) general strategy: "In our view, it is plausible to maintain that in situations such as those in which [the integral conservation law] holds, there exists a quantity in GR that fulfils the functional role of gravitational stress-energy" (pp. 19).

That is, Read adopts the following "functionalism about gravitational energy-stress":

\footnotetext{
${ }^{14}$ For the sake of simplicity, in the remainder of this section $\operatorname{grav} E_{f}$ will denote both the gravitational pseudo-tensor and its charge.
} 


$$
\begin{aligned}
& \left(F U N C_{\text {gravE }}\right) \quad \text { For a quantity } Q \text { to be (represent, ".”") genuine gravitational } \\
& \text { energy-stress is to exhibit a certain profile } \mathcal{F} \text { (gravE) of } \\
& \text { functional roles: } \\
& (\mathcal{F}(\operatorname{gravE}))[Q] \Leftrightarrow Q \doteq \operatorname{gravE}
\end{aligned}
$$

How to flesh out the functional profile of gravitational energy-stress, $\mathcal{F}$ (gravE)? Read determines it to comprise two functional roles:
$(\mathcal{F}($ gravE) $) \quad$ (i) balancing the non-gravitational energy such that the sum is conserved
$\&$
(ii) “(bearing) some relation to the 'gravitational' degrees of freedom in the theory in question" (p. 20).

To complete his argument, a final premise is needed-viz. that $g r a v E_{f}$ plays the preceding two functional roles:

$(\mathcal{F}($ gravE $))\left[\operatorname{grav} E_{f}\right] \quad \operatorname{gravE}_{f}$ instantiates the profile $(\mathcal{F}(\operatorname{gravE}))$.

By construction, $\operatorname{gravE}_{f}$ obeys a (formal) balance equation. Hence, (i) is satisfied. Likewise, (ii) looks harmless: It's customary (e.g. Misner et al. 1973, passim) to identify the metric with the gravitational degrees of freedom (the "gravitational field"); $\operatorname{grav} E_{f}$ is directly and solely built from it.

From the conjunction of $\left(F U N C_{\text {gravE }}\right)$ and $(\mathcal{F}(\operatorname{gravE}))$ now follows that $g r a v E_{f}$ earns the label "gravitational energy". It represents genuine gravitational energy-stress:

$$
\left(F U N C_{\text {gravE }}\right) \&(\mathcal{F}(\operatorname{gravE})) \&(\mathcal{F}(\operatorname{gravE}))\left[\operatorname{gravE}_{f}\right] \rightarrow \operatorname{gravE}_{f} \doteq \operatorname{gravE}
$$

In summary, Read has thus given a formally valid argument for $\left(R E A L_{L O C}\right)$ \& $\left(R E A L_{G L O B}\right)$. Based on the alleged expedience of the gravitational pseudotensor and its associated charge, Read argued for a realist stance towards them. Furthermore, meeting his functional desiderata of gravitational energy, they indeed represent, on his proposal, gravitational energy-stress.

What to make of Read's proposal? Is the appeal to functionalism convincing? Does gravitational energy-stress in GR really satisfy the functional roles, stipulated by Read? Does his proposal overcome the difficulties that undergird Hoefer's eliminativism (Sect. 3.3)? To these questions we now turn. 


\subsection{Objections}

In this subsection, I'll evaluate Read's realism about gravitational energy. Apart from Dennett's Criterion $(D C)$, and the fact that the formal notions of gravitational energy play the two functional roles stipulated by $(\mathcal{F}(\operatorname{gravE}))\left[\operatorname{gravE}_{f}\right]$, I'll question each assumption in his reasoning sketched above.

I'll discuss each premise separately and in increasing order of generality: $(\mathcal{F}$ $(\operatorname{gravE})),\left(F U N C_{\text {gravE }}\right)$ and $(D C)\left[\operatorname{gravE}_{f}\right]$.

\subsubsection{Is Read's functional characterisation of gravitational energy-stress adequate?}

Consider first Read's functional profile of gravitational energy-stress, i.e. $(\mathcal{F}(\operatorname{gravE}))$ : Are the functional roles of gravitational energy-stress adequately characterised by (i) and (ii)? I dispute that: They are neither jointly sufficient nor necessary.

Two facts cast doubt upon the view that (i) and (ii) are jointly sufficient: the triviality of continuity equations, and ambiguity, respectively.

Firstly, formal continuity equations are too easily procurable (Goldberg 1958, p. 17). For any symmetric quantity $\gamma^{\mu \nu}$, one can always construct a symmetric quantity $\Gamma^{\mu \nu}$ that satisfies continuity equation $\partial_{\nu}\left(\sqrt{|g|} T^{\mu \nu}+\Gamma^{\mu \nu}\right)=0-v i z . \Gamma^{\mu \nu}:=\partial_{\varrho, \sigma}$ $\left(\gamma^{\mu \nu} \gamma^{\varrho \sigma}-\gamma^{\nu} \varrho \gamma^{\mu \sigma}\right)-\sqrt{|g|} T^{\mu \nu}$. (Recall that that the energy-stress tensor $T^{\mu \nu}$ also depends on the metric, cf. Lehmkuhl 2011). If one now chooses for $\gamma^{\mu \nu}$ some arbitrary function, e.g. $\gamma^{\mu \nu}=\sin (R) R^{\mu \nu}$, one obtains a quantity that satisfies (i) and (ii). Nonetheless, one would hesitate to ascribe it physical significance as a candidate gravitational energy.

Read may demur at continuity equations thus constructed as they hold irrespective of any field equations (and furthermore that they also depend on the matter degrees of freedom). They are indeed mathematical identities. Read might parry by supplementing (i) with a proviso: the conservation law not be a mathematical identity (and not directly depend on the matter degrees of freedom). ${ }^{15}$

This doesn't alleviate the above worry, though: The previous argument can just be rehashed for $\tilde{\Gamma}^{\mu \nu}:=\partial_{\varrho, \sigma}\left(\gamma^{\mu \nu} \gamma^{\varrho \sigma}-\gamma^{\nu \sigma} \gamma^{\mu \sigma}\right)-\frac{1}{\kappa} \sqrt{|g|} G^{\mu \nu}$. The continuity equation continues to hold-but now in virtue of the Einstein Equations.

Another problem arises from ambiguity. Recall from Sect. 2.3: There exist infinitely many pseudotensors satisfying a local continuity equation. All are built solely from the metric. One needn't even restrict oneself to pseudotensors. Nothing in Read's proposal seems to prevent one from introducing e.g. additional flat background metrics, an orthonormal tetrad or a flat connection (Pitts $2011 \mathrm{~b}$ for a survey of such options). Ditto quasi-local notions (see e.g. Szabados 2009). ${ }^{16}$ Objects with the functional profile $\mathcal{F}$ (gravE) abound.

\footnotetext{
15 This meshes with common practice in the literature on conservation laws: Therein, one distinguishes between "improper" (Hilbert) or "strong" (Bergman) conservation laws on the one hand, and "proper" or "weak" conservation laws on the other (see Brading and Brown 2000; Brading 2005).

However, whether "proper conservation laws" have physical significance eo ipso is a delicate question (Sus 2017). As Read's counter-manoeuvre would arguably seek to ensure physical significance, the proviso would have to be formulated carefully.

16 Quasi-local approaches are plagued by ambiguities of their own, as both Hoefer (2000, p. 196) and Lam (2011, p. 1022) correctly point out.
} 
Unless their mutual consistency can be established, this proliferation of candidate objects that satisfy $\mathcal{F}$ (gravE) should unsettle Read. (Recall our discussion of (R2) in Sect. 3.2.) I therefore conclude: (i)\&(ii) is an insufficient characterisation of the functional profile of gravitational energy.

Further scepticism about the functional roles of $\mathcal{F}$ (gravE) is in order. 1. Conserved quantities are contingent on symmetries. Hence, criterion (i) isn't necessary. 2. Criterion (ii) is bedevilled by general fuzziness, as well as equivocation about the gravitational degrees of freedom.

I'll first argue that (i) imparts a spurious essentiality to a contingent feature of our most familiar spacetime settings.

Underlying Read's stipulation is the intuition that total energy should be conserved. This intuition stems from our habituation to classical theories in flat spacetime (cf. Nerlich 1991). Why expect this to carry over to GR?

The principal motivation stems from the Noether theorems. They establish a general correlation between symmetries of the action and conserved quantities (see e.g. Brading and Brown 2000). Due to its general covariance, GR's action has infinitely many rigid symmetries (see Bergmann 1949, 1958; Brown and Brading 2002; Brading 2005). The Noether Theorems then guarantee, at least formally, infinitely many conservation laws of the pseudotensorial type. To take these formal infinitely many conservation laws seriously, i.e. to regard them as also physically meaningful, leads us back to Pitts' proposal. Whether it deserves realism, remains controversial, as we saw.

One source of reservations about the infinitely many conservation laws may derive from GR's general covariance. Because of the latter, they belong to so-called "improper conservation laws" (Hilbert). These arise from Noether's theorems for all theories with local symmetry group that have a global subgroup (see e.g. Bergmann 1949; Brading and Brown 2000). Their interpretation and physical significance-as Hilbert's label intimates-is subtle: Under certain circumstances, they seem to be (at least, individually ${ }^{17}$ ) trivial, i.e. mathematical identities (see e.g. Brading 2005; Sus 2017), and hence devoid of physical content. What those circumstances exactly are, is a question of current dispute (closely related to the empirical significance of symmetries, see e.g. Kosso 2000; Brading and Brown 2004; Wallace and Greaves 2014; Teh 2015; Murgueitio Ramirez 2019). On a recent proposal (Barnich and Brandt 2002; Sus 2017), GR's improper energy conservation laws can be salvaged from triviality, if the dynamically possible spacetime models considered possess (asymptotic) symmetries. Whether in our world we should take these infinitely many conservation laws seriously, thus depends on whether we should believe that our world instantiates such asymptotic background structure. And indeed, I'll argue below that one should-however, the asymptotic structure is that of a de Sitter space. But that entails two problems. The first is that the integrals of the pseudotensor-based continuity equations diverge. Thereby, the conserved global/integral charges aren't well-defined. But with the symmetries of de Sitter space, also the motivation for a local/differential conservation law, based on pseudotensors, becomes moot: Using the the associated so-called Killing

\footnotetext{
17 The totality of pseudotensorial continuity equations, however, is equivalent to the Einstein field equations (Anderson 1967, p. 427). Hence, it possesses physical significance (cf. Pitts 2009, 2016c).
} 
vectors (see e.g. Read 2018, Sect. 2.4), one can define bona fide (covariant) matter energy-stress fluxes that are covariantly conserved-with no (overt) gravitational contributions (Duerr 2018a, Sect. 2).

The connection with Killing vectors can be developed further along a different direction. Unless the spacetime possesses symmetries (to which special coordinates could be adapted (see e.g. Pooley 2017, sect.), coordinates that would be able to single out pseudotensor-based continuity equations) the pseudotensorial conservation laws thus seem to lack intrinsic meaning. But such spacetime symmetries are contingent: Generic spacetimes lack them; even most do. Why, therefore, cling to energy conservation as a default? It seems more natural to reverse the familiar explanatory asymmetry: Energy conservation, not its failure, needs explanation-in terms of a spacetime's special symmetries (see Carroll 2010 for a slightly brutal way of putting it; cf. Duerr 2018a, Sect. 2). ${ }^{18}$

Of course, one might resist this whole reasoning by pointing to the mathematical fact that, due to general covariance, GR's action has symmetries. But as mentioned before, it's unclear that this, by itself, warrants wider-reaching physical conclusions. (Also bear in mind that that most will hesitate to regard an action as more than a merely auxiliary construct — not a physical quantity. Hence, inferences from its properties to properties of physical systems must be handled with care.)

Let's move on to Read's second functional characteristic of gravitational energy, (ii). It can be opposed for two reasons. One is its vagueness: What exactly is the relation that should hold between a candidate for gravitational energy-stress and the gravitational field?

A second worry is more subtle: What are the gravitational degrees of freedom-the "gravitational field"? ${ }^{19}$ Which quantity represents them, e.g. the metric $g_{\mu \nu}$, the connection coefficients $\Gamma_{\mu \nu}^{\lambda}$ (Einstein's choice, see Lehmkuhl 2014), the Riemann tensor (Synge's choice, Synge 1960), or the deviation from flatness $g_{\mu \nu}-\eta_{\mu \nu}$ (Pooley's choice, Pooley 2013, fn. 20)? ${ }^{20}$ Each choice has some merits in its favour (Lehmkuhl 2008). Read rightly cautions against any premature a priori preference for one.

Yet, it's not obvious that his second functional role for gravitational energy, (ii), can avoid an a priori choice. The pseudotensors in $\left(R E A L_{L O C}\right)$ are the canonical energymomenta associated with the metric as the gravitational field.

Suppose, however, that we identify the connection coefficients $\Gamma_{\beta \gamma}^{\alpha}$ as the gravitational field. Then, the associated canonical energy-stress is the Palatini-pseudotensor

\footnotetext{
18 One may flesh this out further in terms of Strevens '(2011) notion of difference-making.

19 An anonymous referee has voiced misgivings that this isn't a serious question for physics: Although various definitions are possible for the gravitational potential, the proposed choices don't essentially affect the canonical pseudotensor, understood as the canonical energy-stress associated with the gravitational degrees of freedom. To identify the latter, according to her or him, only the gravitational field (whose role is presumably analogous to that of the electromagnetic field) should be used.

I beg to differ. First, arguments from analogy are notoriously defeasible. It's therefore unclear to me that speaking of gravitational potentials is legitimate, let alone illuminating. Secondly, even if we trusted the analogy, how to flesh it out? Which object represents the potential, which the field? For better or worse, we find various options considered in the pertinent physics literature (cf. Lehmkuhl 2008).

20 Read (p. 7 p. 20 , fn. 35) acknowledges this.
} 


$$
\vartheta_{\mu}^{v}[\Gamma]=\frac{\partial \widehat{L}}{\partial\left(\partial_{\nu} \Gamma_{\beta \gamma}^{\alpha}\right)} \partial_{\mu} \Gamma_{\beta \gamma}^{\alpha}-\delta_{\mu}^{\nu} \hat{\mathfrak{L}},
$$

where $\hat{\mathfrak{L}}=\hat{\mathfrak{L}}\left(g_{\mu \nu}, \Gamma_{\mu \nu}^{\lambda}, \partial_{\kappa} \Gamma_{\mu \nu}^{\lambda}\right)$ is the (full) Einstein-Hilbert Lagrangian as a functional of the metric, the connection coefficients, and their first derivatives. ${ }^{21}$ (Note that it satisfies the continuity equation: $\partial_{\nu}\left(\sqrt{|g|}\left(\vartheta_{\mu}^{v}[\Gamma]+T_{\mu}^{v}\right)\right)=0$ with the standard energy-stress tensor $T_{\mu}^{v}$. Again, $\vartheta_{\mu}^{\nu}[\Gamma]$ is determined only up to a superpotential term.)

Being 2nd order in the metric compatible with the connection, $\vartheta_{\mu}^{v}[\Gamma]$ manifestly differs from the Einstein or Landau-Lifshitz pseudotensor, which Hoefer, Lam and Read are considering. What is more, its properties are physically implausible: For instance, it yields divergent integrals for radiating systems (see Murphy 1990 for details).

One may object: By comparing the Einstein pseudotensor and the Palatini pseudotensor, aren't we comparing apples and oranges? The Palatini pseudotensor $\vartheta_{\mu}^{v}[\Gamma]$ is based on the full Einstein-Hilbert Lagrangian-not (as is the Einstein pseudotensor) on the truncated, " $\Gamma \Gamma$ " Lagrangian $\overline{\mathcal{L}}_{(g)}$. If one determines the corresponding Einstein pseudotensor for the full Einstein-Hilbert Lagrangian, both expressions coincide (Novotný 1993).

Prima facie, this is satisfying (and a remarkable property of the Einstein-Hilbert Lagrangian!). Nonetheless, it spells a dilemma for Read. One horn is that Read's criterion seems incomplete: It can't decide between the Palatini pseudotensor and the metric-based pseudotensors. If then, in light of the above considerations, one rules out the former, one thereby has to identify the metric as the gravitational field. (Prima facie this isn't implausible: It surely plays a privileged role. For instance, one cannot write down matter coupling to gravity locally using only a connection. One also needs the metric or something equivalent. ${ }^{22}$ ) But even so, the metric's special status doesn't by itself justify its elevation to the gravitational field - as Read himself admits. ${ }^{23}$

The worry about the right identification of the gravitational field is even more general: Why assume that in GR there exists an ambiguously identifiable gravitational field to begin with? It's not implausible that no choice for the gravitational field is ultimately unique across different contexts (Rey 2013).

In short, Read's second functional role, (ii), on pain of incompleteness, cannot remain neutral on the identification of a gravitational field-against his express intentions.

\footnotetext{
21 The connection needn't be assumed to be metrically compatible ab initio. A variation of $\hat{\mathfrak{L}}$ with respect to both the metric and the connection as independent variables enforces metric compatibility. This variational method is called Palatini approach (e.g. Hobson et al. 2006, Ch. 19.10). The Palatini pseudotensor $\vartheta_{\mu}^{v}[\Gamma]$ naturally emerges within this approach-hence its label.

22 It's worth recalling that also fermions essentially couple to the connection determined by the metric-not any other connection, cf. Pitts, (2012) for details.

23 What about the analogy between GR and Yang-Mills Theory? On the one hand, it would indeed strengthen the identification of the metric as the gravitational field variable. On the other hand, GR isn't a Yang-Mills Theory-at least not in the standard sense (see e.g. Aldrovandi and Pereira 2013, passim). So, from the outset the analogy harbors important subtleties. I therefore side with Read's admonition to caution: What we identify in GR as the gravitational field, requires explicit arguments, and can't be easily read off from the analogy with Yang-Mills Theories.
} 


\subsubsection{Is a functionalist strategy appropriate for gravitational energy-stress?}

Now turn to $\left(F U N C_{\text {gravE }}\right)$ : Why appeal to functionalism in the specific context of gravitational energy in GR? I'll launch two lines of attack against it: First, I'll rebut Read's explicit argument for it; secondly, I'll rehearse the reasons that motivate functionalism in the philosophy of mind, and try to ascertain their analogues.

First, let's examine Read's own argument for a functionalist stance towards gravitational energy. I reject it as unfounded.

What primarily bolsters $\left(F U N C_{\text {gravE }}\right)$ for Read is the sterility of its negation: "[...] the alternative to functionalism is to say that 'the structure of certain DPMs of GR is such that it appears that there exists gravitational stress-energy in those models, but really there is no such stress-energy there'; the payoff to be gained from making such a claim is unclear" (p. 20). In particular, he cautions that without $\left(F U N C_{\text {gravE }}\right)$, one may be barred from potentially more perspicuous avenues for explaining some gravitational phenomena, e.g. binary systems.

I concur with Read on the infertility of dogmatically boycotting higher-level explanations from the outset. Sundry examples from non-gravitational physics attest to that (e.g. Falkenburg 2015; Knox 2016; 2017; Knox and Franklin 2018). Yet, the use of higher-level concepts doesn't per se imply functionalism. ${ }^{24}$ The latter is a specific thesis about the meaning and/or the ontological nature of certain quantities (depending on the strain of functionalism, see below). The purported explanatory pay-off of recourse to gravitational energy-stress as a non-fundamental explanans doesn't per se warrant functionalism about gravitational energy-stress.

Moreover, not even the explanatory pay-off of gravitational energy as a higher-level explanans is obvious. Read concedes that appeal to gravitational energy-stress isn't necessary: "one could indeed explain all general relativistic phenomena, in any model of the theory, simply using the apparatus used to pick out the DPMs of the theory" (p. 20). ${ }^{25}$ The existence of two alternative explanations prompts the question: Which of the two achieves the pay-off that Read extolls? (Contrast this with the case of quasiparticles. Fundamentally, they are collective excitations in a solid. In some regards, they behave like particles. A bottom-up, statistical mechanical treatment would require utopian computational power: We'd have to solve typically $\sim 10^{23}$ coupled differential equations. The pay-off of the higher-level description is manifest.)

\footnotetext{
${ }^{24}$ Read's source of inspiration for functionalism in the philosophy of physics is Wallace (2012), whom he quotes (op.cit, p. 58): "Science is interested with interesting structural properties of systems, and does not hesitate at all in studying those properties just because they are instantiated 'in the wrong way'. The general term for this is "functionalism [...]."

This is a gross simplification of functionalism. Wallace's project is primarily concerned with a realist ontology for higher-level/emergent entities. Functionalism is first and foremost the doctrine that what makes an entity to be of a particular type doesn't depend on the entity's composition. Structural realists -such as Wallace (cf. op. cit., pp. 314) —are eo ipso functionalists about all entities, including higher-level ones. Those with different metaphysical penchants, however, can avail themselves of higher-level explanantia without being functionalists about them.

${ }^{25}$ Schutz (2012, p. 7), for instance, writes: "We know today that it is perfectly possible to describe the generation of gravitational waves and their action on a simple detector without once referring to energy; the quadrupole formula for the generation of the waves and the geodesic equation for their action on a simple detector are all one needs $[\ldots] . . "$
} 
What about binary stars, which Read adduces as an example? The case isn't as clearcut as Read suggests. GR predicts that two stars revolving each other emit gravitational radiation, and increase their orbital frequency. With marvellous accuracy, this has been confirmed (e.g. Will 2014). In line with Read's claim, the standard account indeed involves gravitational (wave) energy as an explanans (cf. e.g. Hobson et al. 2006, Ch. 18): The gravitational wave is supposed to carry away the binary system's total (kinetic plus gravitational) energy; as a result, the stars' orbital frequency increases, with the stars spiralling in towards each other.

In a recent detailed analysis, however, Duerr (2018b) compares this standard interpretation of the binary stars to the alternative without gravitational (wave) energy which Read adumbrates. The latter is found to trump the former on the four explanatory virtues of parsimony, scope, depth, and unificatory power. At least pro tempore, this diminishes the force of Read's argument, or even shifts the burden of proof upon Read's shoulders. ${ }^{26}$

Two caveats are in order. First, examples might eventually be found in favour of Read's claim (e.g. in a similar analysis of instabilities in rotating neutron stars, induced by gravitational radiation, see e.g. Schutz and Ricci 2010, Sect. 6.2). ${ }^{27}$ But for the dialectic of the debate to progress, detailed case-studies of such examples are needed. At the moment, they aren't available. Secondly, some of the persuasiveness of Duerr's (2018a, b) arguments depends on whether one shares his GR-exceptionalist creed (see Sect. 1). But Read gives no explicit reasons for or against it.

My second line of attack against ( $F U N C_{\text {gravE }}$ ) adverts to the motivation for functionalism in the philosophy of mind. I submit, it doesn't carry over to the case at hand.

The functionalism, which Read (via Wallace) imports into the philosophy of physics, stems from the philosophy of mind (see e.g. Van Gulick 2009; Levin 2013; Braddon-Mitchell and Jackson 2007, Part I, II, IV). It's mainly motivated by two dif-

\footnotetext{
${ }^{26}$ It's possible to gainsay this conclusion, and still uphold an argumentative asymmetry in favour of Read's position. According to the pragmatic account of explanation, developed by Van Fraassen (1980), what counts as a good explanation is always relative to a particular context. From this angle, $I$ seem to make the stronger claim that there is no context in which explanatory recourse to gravitational energy-stress pays off. Therefore, it would appear incumbent on $m e$ to corroborate it. Moreover, the heuristic and didactic benefits seem obvious - not least since appeal to gravitational energy is an almost undisputed practice in the physics literature.

On the one hand, I readily acknowledge some merits of explanations with gravitational energy in certain contexts. On the other hand, the above counter doesn't sway me for two reasons. Firstly, Read would be ill-advised to be wedded to one particular - invariably controversial-account of explanation (cf. e.g. Woodward 2014). Secondly, the conjunction of the context-relativity of explanations, and Dennett's Criterion entails an outré context-relative ontology. If what counts as a successful explanans depends on the context, and if the role that a successful explanans plays determines what the explanans is, it depends on the context what constitutes a successful explanans. Combine this now with (DC): Successful explanantia merit realist commitment. A kaleidoscopic ontology ensues: The world is populated with a plethora of motley entities; depending on the context, what exists in the world would vary. This lack of coherence strikes me as unpalatable in an ontology.

27 The most promising place for such an argument is arguably black hole thermodynamics. I forgo the topic for two reasons. Firstly, the current paper's ambit is classical GR. I steer clear of any non-classical/quantum aspects. Secondly, the status of black hole thermodynamics is the current topic of dispute, cf. Dougherty and Callender (2016) and Wallace (2017). Hence, it's unclear what inferences to draw from the putative significance of gravitational energy for it.
} 
ficulties: the non-intersubjectivity of mental states, and the identity theory's failure to account for multi-realisability, respectively.

The first is a general and epistemological point: We can't directly know other people's mental states. They defy inter-subjectivity: A tooth-ache is inherently "private". At best, we can infer mental states indirectly from external indicators (screams, tears, etc.). If thus we want to attribute mental states to other people, prima facie we have to postulate them as entities whose intrinsic nature is elusive. (Mental states might - at best-be accessible introspectively. ${ }^{28}$ ) It's sound philosophical advice to strive to minimise the gap between our speculations about the world and our knowledge. How then to accommodate for mental states?

A second motivation for functionalism arises from a shortcoming of the preceding identity theory. According to the latter, mental states (or properties) are identical with physical states (or properties). Mental states are multiply realisable: It seems unduly chauvinistic to decree apriori that organisms can't be ascribed the same (or sufficiently similar) mental states, despite neuroanatomical and neurophysiological differences. Why shouldn't, say, Read and an octopus both be able - at least in principle - to experience pain and pleasure? But on the identity theory it remains mysterious, how two intrinsically sufficiently different brain states can be identical with the same mental state.

Both difficulties can be eschewed by characterising mental states not via intrinsic properties of brain states, but via their function: They are individuated by the structural roles they play in a (neuronal) network.

Do these two motivations have counterparts for the case of gravitational energy-stress in GR? Three disanalogies speak against it: its absence in the manifest image, its non-privacy and absence of multi-realisability.

First, on the one hand, gravitational energy-stress - unlike mental states - isn't an empirical phenomenon that needs to be accounted for. On the other hand, unlike (say) belief states, even as a theoretical concept, gravitational energy scarcely counts as a robust folk-theoretic notion in our manifest image that an adequate scientific theory in one way or the other must save. ${ }^{29}$ Read himself acknowledges that it's-at least conceivably—dispensable.

\footnotetext{
28 Dennett (1991b) denies even that.

${ }^{29}$ Herein lies a key difference to other areas in philosophy of physics where functionalist strategies are deployed.

Consider first Everettian quantum mechanics. One of its major challenges is how to recover our manifest image of macro-objects in 3-dimensional space, like crystals and anteaters, from the scientific image of a single, richly structured entity, defined on a higher-dimensional so-called configuration space. The appearance of three-dimensionality is a robust phenomenon that on pain of empirical incoherence arguably needs to be accounted for (e.g. Ney 2010). To achieve this, Everettians routinely appeal to functionalism (e.g. Wallace 2012, Ch. 2; Ney forth.).

Another example are quantum theories of gravity (cf. Le Bihan forth. for a detailed analysis between functionalism in the philosophy of mind and philosophy of quantum gravity) devoid of familiar (e.g. smooth) spatiotemporal structure (Lam and Wüthrich 2018). Given that the latter is a robust phenomenon, one must arguably be able to give a story of how to recover it from our "a-spatiotemporal" scientific image.

Notice also that, by contradistinction, Knox's "inertial frame functionalism" clearly doesn't aim at recovering the manifest image. In this regard, then, Wallace's and Lam and Wüthrich's projects are closer to functionalism in the philosophy of mind than are Knox's or Read's respective uses.
} 
Secondly, being a physical quantity, gravitational energy doesn't suffer from the privacy of mental states: Nobody is endowed with a privileged introspective access to gravitational energy-stress, opaque to lesser mortals.

A less quirky sense of "privacy" in this context takes its cue from Dennett (1991a, b, cf. Ladyman and Ross 2007, pp. 161). ${ }^{30}$ For him, it's typical of real patterns to become visible only on higher-levels of description. On the fundamental level, one may lose their salience out of sight: One doesn't see the wood for the trees. (This is the sense in which the higher-level explanations, discussed by Knox $(2016,2017)$ and Knox and Franklin (2018) reveal the salient features, otherwise opaque on the microphysical level.)

Is gravitational energy "private" in this sense? Can it only be properly understood on the coarse-grained, higher-level which Read's functionalist perspective envisions? That, too, I impugn. Formal notions of gravitational energy aren't higher-level concepts in the relevant sense: They are non-fundamental in that they are only definable in certain subclass of models. Again, the motivation from "privacy" founders.

Thirdly, multi-realisability has no obvious counterpart. Recall that it's an interlevel relationship: It links higher-level and lower-level (more fundamental) entities. $\left(F U N C_{\text {gravE }}\right)$ presupposes that the functional profile of gravitational energy is supplied from gravitational theories other than GR.

The most straightforward such "reference theory" is Newtonian Gravity. GR reduces to it in the weak gravity limit. ${ }^{31}$ Hence, the functional role would be fixed by GR itself in a particular regime. (One may already ponder: Isn't it ad-hoc to accord an ontological privilege to this particular regime? The more modest goal of identifying rough-and-ready functional counterparts of quantities in antecedent theories is, of course, harmless. See below.) Suppose now that in another regime, GR exhibits some structural similarity to the weak-field regime. This similarity doesn't constitute an inter-level relationship of the kind required for multi-realisability. It doesn't link a fundamental and a less fundamental level of description. Rather it's an intra-level relationship. The same applies to different reference theories, say massive graviton gravity. ${ }^{32}$ Both GR and it vie for providing the best description of the same domain. They operate on the same ontological level. Again, we aren't dealing with multirealisability.

It's terminological confusion to say that GR "instantiates" or "realises" some quantity, defined in massive graviton gravity. Of course, one could meaningfully ask: "What structures of a GR spacetime are the (rough) analogues or counterparts of some quan-

\footnotetext{
30 I thank James Read (Oxford) for alerting me to this possibility.

31 Herein lies another, albeit arguably peripheral, difference to the philosophy of mind: It's not even clear how best to conceptualise a potential reduction of the mental to the physical-let alone whether it can successfully be carried out (cf. e.g. Beckermann 2008, Ch. 8,9).

32 Massive spin-2 graviton theory (e.g. Hinterbichler 2012; de Rham 2014) happens to be empirically adequate for suitable field masses (Pitts and Schieve 2007; Pitts 2011a, 2016a, b, c).
} 
tity in massive graviton gravity?"33 But gleaning structural similarities is ontologically much less ambitious than Read's realism. ${ }^{34}$

In short: The two main motivations for functionalism in the philosophy of mind-non-intersubjectivity of mental states and multi-realisability-lapse for gravitational energy-stress. This corrodes any tangible motivation for $\left(F U N C_{g r a v E}\right)$.

This conclusion calls for qualification. Plausibly, the above motivations are (individually) sufficient conditions for the application of functionalism. I don't claim that they are necessary. But to my knowledge, there aren't any other motivations for functionalism in the literature. Hence, it seems not unfair to request of Read a justification of his functionalist strategy, should it be motivated "non-standardly".

\subsubsection{Is Dennett's Criterion really satisfied?}

Let's eventually revert to Read's reality criterion, (DC). To decide whether the formal concepts of gravitational energy, gravE $_{f}$, capture real structures, Read employs Dennett's reality criterion (DC): If a higher-level quantity is well-defined and explanatorily/predictively useful, it merits realist commitment. Are the antecedent conditions really satisfied?

Let's hark back to the main finding of our more careful exegesis of Hoefer's first objection, $(H 1)$, in Sect. 3: Realism about local and global pseudotensorial gravitational energy-stress, $\left(R E A L_{L O C}\right)$ and $\left(R E A L_{G L O B}\right)$, is obstructed both by the pseudotensor's ambiguity/non-uniqueness and vicious coordinate-dependence (unless Read's position collapses onto Pitts'-a position for which Read gives no independent arguments). Read's responses to Hoefer were seen to be either ineffective or incomplete. His functional approach discussed added nothing relevant as regards these problems: The first antecedent condition of $(D C)$ isn't satisfied: Gravitational energy-stress isn't well-defined (except for Pitts' object).

What about the other condition-explanatory utility? Read still owes us an argument, or full-fledged example, for why gravitational energy-stress is a powerful

\footnotetext{
33 "Analogue gravity" illustrates this. Some non-gravitational systems, e.g. Bose-Einstein condensates or sound waves in relativistic fluids, can simulate certain features of general-relativistic gravity (see e.g. Visser et al. 2011). Rather than instances of multiple realisations of (general-relativistic) gravity, they merely provide insights by exploiting analogical structures (cf. Dardashti et al. 2015 for a philosophical analysis).

34 This is how I classify the "spacetime functionalism", promulgated by Knox (2013, ms, 2017a, b): It uses the functional role of GR's spacetime to identify the counterparts of (i.a.) GR's inertial structure in other spacetime theories. This is an application of Lewis' $(1970,1972)$ proposal of functional definitions. Via the latter, one determines correspondences between theoretical terms of two theories rather than (Nagelian) reductions via bridge laws.

In other words: Knox's "spacetime functionalism", to my mind, is a form of "commonsense functionalism", which uses the functional role as a reference-fixing device (cf. Braddon-Mitchell and Jackson 2007, Ch. 3, 15). I regard it as the strength of Knox's position that it's not inherently committed to anything ontologically more ambitious (e.g. regarding this functional role as constituting the essence of spacetime).

In particular, my reading of Knox's spacetime functionalism as a "commensense functionalism" is congenial to Baker (2019), who argues that spacetime is a cluster concept: Spacetime structure in various theories plays many roles, with no single role being sufficient or necessary for spacetime simpliciter. This is exactly what to expect of correspondences between terms belonging to distinct theories: Usually, there are no unique, one-to-one correspondences.
} 
explanans. ${ }^{35}$ To my mind, this can only be satisfactorily gauged through detailed case studies (e.g. of energy extraction processes in Black Holes, see e.g. Geroch 1973). Nonetheless, a strong argument for eliminativism can already be made, turning on a wide range of astrophysical and cosmological phenomena.

Recall that Read's $\left(R E A L_{G L O B}\right)$ hinges on realism about asymptotic flatness. In Sect. 3.3, I suggested that the disagreement between Hoefer and Read over the acceptability of asymptotic flatness best be understood as a disagreement between different classifications: Whereas for Read asymptotic flatness is a good approximation, for Hoefer it's an idle posit in an idealisation. The bone of contention is therefore: Do the salient features of empirically confirmed asymptotically flat models successfully refer? Primarily in light of contemporary cosmology, I contend, they don't.

On the one hand, many spacetimes utilised for modelling the exterior of stationary astrophysical objects are indeed asymptotically flat. Apart from the Schwarzschild metric, the the Kerr-Newmann solution for the exterior of a rotating, charged black hole is a case in point (cf. Reiris 2014 for a proof of a large class of spacetimes). But unfortunately, no interior solution for the (uncharged) Kerr metric is known whose source is a perfect fluid-the simplest model for a star.

This may merely be regrettable (perhaps even a temporary issue). But more generally, Christodoulou and Klainerman (1993, p. 10) warn: “[...] (I)t remains questionable whether there exists any nontrivial (non-stationary) solution of the field equations that satisfies the Penrose requirements [i.e. the geometric conditions encoding asymptotic flatness]. Indeed, his regularity assumptions translate into fall-off conditions of the curvature that may be too stringent and thus may fail to be satisfied by any solution that would allow gravitational waves. Moreover, the picture given by the conformal compactification fails to address the crucial issue of the relationship between the conditions in the past and in the behaviour in the future."

The only known non-stationary, asymptotically flat solutions (e.g. within the Robinson-Trautman class of metrics describing expanding gravitational waves) are marred by singularities. This threatens their physicality.

There are two responses to this. One is that singularities may not be as calamitous as orthodoxy (e.g. Earman 1995, p. 12) has it (Curiel and Bokulich 2009, Sect. 2; Lehmkuhl 2017). Another reaction points to approximate solutions based on perturbative methods. Via them one can determine the spacetime of, say, an in-spiralling compact binary system, yielding a spacetime that is non-stationary and asymptotically flat.

This leads us to the major objection to asymptotic flatness as an approximation-cosmology. Prior to that, though, let's briefly dwell on the perturbative approximation schemes featuring in the treatment of binary systems. In a nutshell (see e.g. Maggiore 2007, Ch. 5; Poisson and Will 2014 for details), in the astrophysical system's neighbourhood, one employs the so-called Post-Newtonian approximation scheme-an expansion in powers of a small parameter $\left(1 / c^{2}\right)$ - to determine the system's near field. But this expansion in the near-zone expansion is a singular perturbation theory: For distances tending to infinity, higher-order terms blow up; the Post-Newtonian

\footnotetext{
35 Note that Dennett (1989, Ch. 2, 3; 1991a, 2009) accentuates the importance of the immense gain in explanatory power-nigh-universal scope and practical ineliminability — for the bona fide applications of his criterion.
} 
scheme isn't uniformly valid for all distances. In particular, it cannot incorporate the no-incoming radiation boundary conditions, apt for gravitationally radiating objects. One therefore adopts a different approximation scheme for the so-called "far-field zone". In the intermediate region, both expansions are then smoothly glued together ("matched asymptotic expansion"). Which boundary conditions to impose for the far-field zone? A standard choice is asymptotic flatness.

Here lies the principal reason for classifying asymptotic flatness as an idealisation: According to today's best cosmological model, we live in an FLRW universe with a positive cosmological constant $\Lambda$. It leads to infinite (albeit ever slower) expansion in our universe's long-term future: Our universe is asymptotically deSitter; it's not asymptotically flat (see e.g. Carroll 2003; Rubin and Hayden 2016 for details).

Already for the exterior of the simplest, i.e. spherically symmetric star model, immersed in a deSitter spacetime, asymptotic flatness breaks down. Does this vitiate all-well-confirmed! — calculations based on an asymptotically flat far-field? Luckily_no: Far from the source, but still much closer than cosmological scales, spacetime is approximately flat - for all practical purposes. So, the usual techniques apply-as long as one doesn't venture too "far out" in space and time (Ashtekhar et al. 2016; Bonga and Hazboun 2017).

Asymptotic flatness is therefore an idealised extrapolation of the ambient spacetime at a particular phase of a star's life: One ignores its future beyond a certain point, prescinding from the star's cosmic embedding. The referents of asymptotically flat spacetimes are therefore ahistorical fictional objects. The practising physicist uses them as convenient surrogates for the real target objects, e.g. a pulsar, a galaxy, etc., because they share with the latter the relevant structural features up to cosmological scales. (It's this omission of actual history that physicists mean, when taking asymptotic flatness to characterise isolated systems. An object in an asymptotically flat spacetime is dynamically isolated in the sense that it quiesces into a stationary state. ${ }^{36}$ ) Asymptotically flat spacetimes thus are idealisations. Even when successful, they describe surrogate systems, distinct (with respect to their past or future evolution) from remotely physical ones.

More importantly, the working posits of successful asymptotically flat models aren't their fall-off behaviour at infinity. Rather, they are the right fall-off behaviour up to cosmological scales: All empirical content is garnered from the properties of a finite patch of an asymptotically flat spacetime. But it's, of course, the behaviour at infinity that is salient of asymptotic flatness. Asymptotic flatness is therefore an idle posit. Recourse to $(D C)$ is thus blocked.

In short: Gravitational energy in Read's proposal contravenes both conditions of Dennett's Criterion. Owing to its coordinate-dependence and ambiguity, local and global gravitational energy is ill-defined (unless Read's position collapses onto Pitts', for which then he should argue explicitly). Moreover, asymptotic flatness is an idle posit. Hence, it doesn't yield the explanatory mileage that a realist would urge.

I conclude that Read's argument for a realism about pseudotensor-based global and local gravitational energy fails. In consequence, vis-à-vis Read's proposal, Hoefer's

\footnotetext{
36 I propose that Nerlich's main argument (2013, pp. 159) should be understood (more charitably) along these lines: Asymptotic flatness imposes a stationary long-term future-contrary to our best cosmological knowledge.
} 
alternative seems preferable. It cuts the Gordian knot: We should indeed be eliminativists about gravitational energy, and recognise that in GR, energy just ceases to be conserved as a default (see Schroedinger 1950, p. 105 for a "singularly striking example", cf. Misner et al. 1973, Sect. 19.4).

\section{Outlook}

While I argued that Read's proposal should be rejected, his general functionalist approach to gravitational energy can be salvaged, and prove fecund in two slightly different contexts. For that, though, we must be clear on what it is: A scheme that allows us in a principled manner to

1. assess when a (cautious) realist stance towards certain non-fundamental quantities is apposite-via Dennett's Criterion;

2. identify those as counterparts of Newtonian gravitational energy in other theories-via Lewisian functional definitions.

One such promising context concerns non-pseudotensorial approaches to global gravitational energy-stress; the other concerns a research programme inaugurated recently by Ashtekar and collaborators.

Whilst Read doesn't mention them, three other candidates for global gravitational energy lend themselves to his agenda (as I believe, it ought to be understood): the Komar mass, the Bondi-Sachs mass, and the ADM mass. Being non-pseudotensorbased, they circumvent the two main defects of pseudotensors discussed above. To gauge the prospects, I'll comment on each. (I'll skip the technical details. For them, I refer to Wald 1984, Ch. 11.2; Poisson 2004, Ch. 4.3; Jaramillo and Gourgoulhon, Ch. 3 , and references therein.)

Start with the Komar mass. I submit, it violates either the first or the second antecedent conditions of Dennett's Criterion.

For stationary (and asymptotically flat) spacetimes, it furnishes a well-defined, coordinate-independent notion of global gravitational energy. But this augurs only a Pyrrhic victory for Read. The casualty is physical significance: Stationarity precludes astrophysical processes like stellar evolution, gravitational or electromagnetic radiation. Realism about the Komar mass thus is Pickwickian: Its limited applicability is at variance with any demand for explanatory utility, i.e. the second condition of (DC).

The only spacetimes capable of describing in any sense realistic systems, and hence the only ones capable of empirical confirmation, are of course non-stationary. But for non-stationary, asymptotically flat spacetimes, the Komar energy requires a gauge-fixing in the following sense: For the integral to be well-defined, a coordinate condition needs to be imposed on the representative of the equivalence class of socalled Bondi-Metzner-Sachs time translations. (Loosely speaking, the latter encode translations at infinity, see e.g. Wald 1984, pp. 283 for details.) This gauge-fixing amounts to privileging a certain subclass of time-translations. (NB: The problem isn't the imposition of a coordinate condition per se. Rather it's the fact that thereby one singles out time-translations along directions that aren't intrinsically distinguished.) This looks like a drastic, if not ad-hoc restriction of the concept of energy. It thus 
seems that for empirically relevant contexts, the Komar mass violates the demand for well-definedness, i.e. the first condition of (DC).

A second approach to gravitational energy is the Bondi-Sachs mass. For asymptotically flat spacetimes, it's defined as an integral at "null infinity". Roughly speaking, that is: One evaluates the solution-valued Hamiltonian of GR in the limit surface at infinity along the light cone. (Equivalently, one can conceive of the Bondi-Sachs quantities as Noether charges, associated with the symmetries of asymptotically flat spacetimes at null infinity.) The Bondi mass captures the energy that electromagnetic or gravitational radiation carries off to infinity. In the presence of an outward energy flux, the Bondi-Sachs mass decreases. But it always remains non-zero. It's also bounded from above by the third candidate for gravitational energy in asymptotically flat spacetimes- the ADM mass.

In contrast to the Bondi-Sachs mass, it's defined at "spatial infinity": One evaluates the solution-valued GR Hamiltonian in the limit of spacelike hypersurfaces stretching to infinity. The ADM mass is a suitable candidate for total energy-momentum of spacetime. By construction, it's conserved. A celebrated result of mathematical physics is that the ADM mass can be shown to be positive (for matter satisfying certain energy conditions). Furthermore, under suitable conditions, it initially coincides with the Bondi-Sachs mass. Accordingly, the latter can be interpreted as the residual ADM energy after gravitational and electromagnetic wave energy has been extracted from the system.

What to make of the Bondi-Sachs and ADM mass in the present context? The reflections on the status of asymptotic flatness as an idealisation in Sect. 4.3.2 curtail rash hopes: With asymptotic flatness as their prerequisite (Jaramillo and Gourgoulhon 2010) both the Bondi-Sachs and ADM mass don't seem to merit realist commitment.

In short, Read's functionalism about gravitational energy doesn't fare significantly better for the three standard non-tensorial notions of global gravitational energy. The Komar mass is either ill-defined or deficient in explanatory power. Both the (standard) Bondi-Sachs and the ADM mass presuppose asymptotic flatness. With the latter being an idle posit of an idealisation, neither seems to merit realist commitment.

Another context, however, deserves greater attention. In it, Read's proposal (understood as sketched above, and with the suitable amendments with respect to the characterisation of the functional profile of gravitational energy) may prove valuable: the framework for asymptotic structure of spacetimes with a cosmological constant, $\Lambda>0$, recently developed in a series of papers by Ashtekar et al. (2015a, b, c). It promises to circumvent some of the problems diagnosed for Read's approach. In particular, given that our universe is arguably asymptotically deSitter, the asymptotic structure on which Ashtekar et al.'s framework relies may well count as a working posit of an approximation. It remains to be seen whether the symmetries of deSitter space allow for a satisfactory formal definition of gravitational energy — and what the functional roles are that it plays. But supposing that gravitational energy does admit of a well-defined expression in this context, prima facie it's a counterpart of Newtonian gravitational energy, about which we should be realists.

Acknowledgements I am indebted to James Read (Oxford), Juliusz Doboszewksi (Bonn), Ahmad Elabbar (Cambridge), and Oliver Pooley (Oxford) for extensive, insightful feedback: sincere thanks! Also a big 
thank you to Brian Pitts (Cambridge) for many inspiring and instructive conversations. This research was generously supported by a doctoral scholarship of the British Society for the Philosophy of Science, and the Heinrich Hertz scholarship in History and Philosophy of Physics (Bonn).

Open Access This article is licensed under a Creative Commons Attribution 4.0 International License, which permits use, sharing, adaptation, distribution and reproduction in any medium or format, as long as you give appropriate credit to the original author(s) and the source, provide a link to the Creative Commons licence, and indicate if changes were made. The images or other third party material in this article are included in the article's Creative Commons licence, unless indicated otherwise in a credit line to the material. If material is not included in the article's Creative Commons licence and your intended use is not permitted by statutory regulation or exceeds the permitted use, you will need to obtain permission directly from the copyright holder. To view a copy of this licence, visit http://creativecommons.org/licenses/by/4.0/.

\section{References}

Aldrovandi, R., \& Pereira, J. G. (2013). Teleparallel gravity: An introduction. Heidelberg: Springer.

Anderson, J. L. (1967). Principles of relativity physics. New York: Academic Press.

Anderson, J. L. (1971). Covariance, invariance, and equivalence: A viewpoint. General Relativity and Gravitation, 2, 161.

Ashtekhar, A., Bonga, B., \& Kesevan, B. (2015a). Asymptotics with a positive cosmological constant: I. Basic framework. Classical and Quantum Gravity, 32, 025004. https://doi.org/10.1088/0264-9381/ 32/2/025004/.

Ashtekhar, A., Bonga, B., \& Kesevan, B. (2015b). Asymptotics with a positive cosmological constant: II. Linear fields on de Sitter space-time. Physical Review D, 92, 044011. https://doi.org/10.1103/ PhysRevD.92.044011.

Ashtekhar, A., Bonga, B., \& Kesevan, B. (2015c). Asymptotics with a positive cosmological constant: III. The quadrupole formula. Physical Review D, 92, 10432. https://doi.org/10.1103/PhysRevD.92. 104032.

Ashtekhar, A., Bonga, B., \& Kesevan, B. (2016). Gravitational waves from isolated systems: Surprising consequences of a positive cosmological constant. Physical Review Letters, 116, 051101. https://doi. org/10.1103/PhysRevLett.116.051101.

Baker, D. (2019). On spacetime functionalism. http://philsci-archive.pitt.edu/15860/. Accessed Nov 2019.

Barbashov, B. M., \& Nesterenko, V. V. (1983). Continuous symmetries in field theory. Fortschritte der Physik (Progress in Physics), 31(10), 535-567. https://doi.org/10.1002/prop.2190311003.

Barnich, G., \& Brandt, F. (2002). Covariant theory of asymptotic symmetries, conservation laws and central charges. Nuclear Physics B, 633, 3D82. https://doi.org/10.1016/S0550-3213(02)00251-1.

Beckermann, A. (2008). Analytische Einführung in die Philosophie des Geistes. Berlin: de Gruyter.

Bergmann, P. G. (1949). Non-linear field theories. Physical Review, 112, 287. https://doi.org/10.1103/ PhysRev.75.680.

Bergmann, P. G. (1958). Conservations in general relativity as the generators of coordinate transformations. Physical Review, 112, 287.

Bonga, B., \& Hazboun, J. (2017). Power radiated by a binary system in a de Sitter Universe. Physical Review D. https://doi.org/10.1103/PhysRevD.96.064018.

Braddon-Mitchell, D., \& Jackson, F. (2007). Philosophy of mind and cognition. Malden, MA: Blackwell Publishing.

Brading, K. (2005). A note on general relativity, energy conservation, and Noether's theorems. In A. J. Kox \& J. Eisenstaedt (Eds.), The universe of general relativity. Einstein studies: 11. Boston: Birkhaeuser.

Brading, K., \& Brown, H. R. (2000). Noether's theorems and gauge symmetries. Diálogos 79, 59-86. Preprint: https://arxiv.org/abs/hep-th/0009058.

Brading, K., \& Brown, H. R. (2004). Are gauge symmetry transformations observable? British Journal for the Philosophy of Science, 55, 645-665. https://doi.org/10.1093/bjps/55.4.645.

Brown, H. (2005). Physical relativity. Relativity from a dynamical perspective. Oxford: Oxford University Press.

Brown, H., \& Brading, K. (2002). General covariance from the perspective of Noether's theorems. http:// philsci-archive.pitt.edu/821/. Accessed Nov 2019. 
Carroll, S. (2003). Why is the universe accelerating? In: W. L. Freedman (Ed.), Carnegie observatories astrophysics series, Vol. 2: Measuring and modeling the universe. Cambridge: Cambridge University Press. Preprint: https://arxiv.org/abs/astro-ph/0310342.

Carroll, S. (2010). http://www.preposterousuniverse.com/blog/2010/02/22/energyis-not-conserved/. Accessed Nov 2019.

Christodoulou, D., \& Klainerman, S. (1993). The global nonlinear stability of the Minkowski space. Princeton: Princeton University Press.

Curiel, E. (2000). The constraints general relativity places on physicalist accounts of causality. Theoria, $15(1), 33-58$.

Curiel, E. (2018). On geometric objects, the non-existence of a gravitational stress-energy tensor, and the uniqueness of the Einstein field equation. https://arxiv.org/pdf/1808.08998.pdf.

Curiel, E., \& Bokulich, P. (2009). Singularities and black holes. Stanford encyclopaedia of philosophy. https://plato.stanford.edu/entries/spacetime-singularities/\#BreGenRel. Accessed Nov 2019.

Dardashti, R., Thébault, K., \& Winsberg, E. (2015). Confirmation via analogue simulation: what dumb holes could tell us about gravity. British Journal for the Philosophy of Science, 68(1), 55-89. https:// doi.org/10.1093/bjps/axv010.

de Rham, C. (2014). Massive gravity. Living Reviews in Relativity. https://doi.org/10.12942/lrr-2014-7.

Dennet, D. (1991). Consciousness explained. Boston: Little, Brown \& Co.

Dennett, D. (1989). The intentional stance. Cambridge: MIT Press.

Dennett, D. (1991). Real patterns. Journal of Philosophy, 88(1), 27-51.

Dennett, D. (2009). Intentional systems theory. In B. McLaughlin, A. Beckermann, \& S. Walter (Eds.), Oxford handbook to the philosophy of mind (p. 2009). Oxford: Oxford University Press.

Dougherty, J., \& Callender, C. (2016). Black hole thermodynamics: more than an analogy? In: A. Ijjas, \& B. Loewer (eds.). Guide to the philosophy of cosmology. Oxford: Oxford University Press. Preprint: http://philsci-archive.pitt.edu/13195/. Accessed Nov 2019.

Duerr, P. (2018a). Fantastic beasts and where (not) to find them: Energy conservation and local gravitational energy in general relativity. Studies in History and Philosophy of Modern Physics. https://doi.org/10. 1016/j.shpsb.2018.07.002.

Duerr, P. (2018b). It aint't necessarily so: Gravitational waves and energy transport. Studies in History and Philosophy of Modern Physics. https://doi.org/10.1016/j.shpsb.2018.08.005.

Earman, J. (1995). Crunches, whimpers and shrieks: Singularities and acausalities in relativistic spacetimes. Oxford: Oxford University Press.

Eddington, A. (1923). The mathematical theory of relativity. Cambridge: Cambridge University Press.

Einstein, A. (1918). Der Energiesatz in der allgemeinen Relativitätstheorie. Sitzungsberichte der Königlich Preußischen Akademie der Wissenschaften (Berlin), Seite 448-459.

Falkenburg, B. (2015). How do quasi-particles exist? In B. Falkenburg \& M. Morrison (Eds.), Why more is different Philosophical issues in condensed matter physics and complex systems. Heidelberg: Springer.

Feynman, R. (1995). Feynman lectures on gravitation. Boulder: Westview Press.

Friedman, M. (1983). Foundations of space-time theories. Relativistic physics and philosophy of science. Princeton: Princeton University Press.

Geroch, R. (1972). General relativity. 1972 Lecture Notes. Montreal: Minkowski Institute Press, 2016 (reprint).

Geroch, R. (1973). Energy extraction. http://strangebeautiful.com/other-texts/geroch-energy-extraction. pdf. Accessed Nov 2019.

Goldberg, J. N. (1958). Conservation laws in general relativity. Physical Review, 111(1958), 315-320. https://doi.org/10.1103/PhysRev.111.315.

Gorelik, G. (2002). The problem of conservation laws and the Poincare quasigroup in general relativity. In Y. Balashov \& V. Vizgin (Eds.), Einstein studies in Russia (pp. 17-43). Boston: Birkhaeuser.

Hettema, H. (2012). Reducing chemistry to physics: Limits, models, consequences. PhD thesis, University of Groningen.

Hinterbichler, K. (2012). Theoretical aspects of massive gravity. Reviews of Modern Physics, 84, 671-710.

Hobson, M. P., Efstathiou, G. P., \& Lasenby, A. N. (2006). General relativity: An introduction for physicists. Cambridge: Cambridge University Press.

Hoefer, C. (2000). Energy conservation in GTR. Studies in History and Philosophy of Modern Physics, 31(2), 187-199.

Horský, J., \& Novotný, J. (1969). Conservation laws in general relativity. Czechoslovak Journal of Physics, 19(419), 1969. 
Isenberg, J. (2014). The initial value problem in general relativity. In A. Ashtekar \& V. Petkov (Eds.), Springer handbook of spacetime (p. 2014). Dordrecht: Springer.

Janssen, M. (2009). Drawing the line between kinematics and dynamics in special relativity. Studies in History and Philosophy of Modern Physics, 40, $26 \mathrm{e} 52$.

Jaramillo, J. L., \& Gourgoulhon, E. (2010). Mass and angular momentum in general relativity. In L. Blanchet, A. Spallicci, \& B. Whiting (Eds.), Mass and motion in relativity (p. 2010). Heidelberg: Springer.

Kaiser, D. (1998). A $\psi$ is just a $\psi$ ? Pedagogy, practice, and the reconstitution of general relativity, 1942-1975. Studies in History and Philosophy of Modern Physics, 29(3), 321-338. https://doi.org/ 10.1016/S1355-2198(98)00010-10.

Katz, J. (1985). A note on Komar's anomalous factor. Classical and Quantum Gravity, 2, 423.

Katz, J. (2005). Gravitational energy. Classical and Quantum Gravity, 22(5169), 2005.

Katz, J., Bičak, \& Lynden-Bell, D. (1997). Relativistic conservation laws and integral constraints for large cosmological perturbations. Physical Review D, 55(5957), 1997.

Knox, E. (2013). Effective spacetime geometry. Studies in History and Philosophy of Modern Physics., 44(3), 2013. https://doi.org/10.1016/j.shpsb.2013.04.002.

Knox, E. (2016). Abstraction and its limits: finding space for novel explanations. Noûs, 50(1), 41-60. https://doi.org/10.1111/nous.12120.

Knox, E. (2017a). Novel explanations in the special sciences: Lessons from physics. http://philsci-archive. pitt.edu/13406/. Accessed Nov 2019.

Knox, E. (2017b). Physical relativity from a functionalist perspective. Studies in History and Philosophy of Modern Physics, forth.. https://doi.org/10.1016/j.shpsb.2017.09.008.

Knox, E. (ms). Spacetime functionalism or spacetime structuralism (unpublished manuscript), https:// www.eleanorknox.com/uploads/3/9/8/2/39826525/structuralism_or_functionalism_draft_2.pdf.

Knox, E., \& Franklin, A. (2018). Emergence without limits: The case of phonons. Studies in History and Philosophy of Modern Physics. https://doi.org/10.1016/j.shpsb.2018.06.001.

Kosso, P. (2000). The empirical status of symmetries in physics. British Journal for the Philosophy of Science, 51, 81-98. https://doi.org/10.1093/bjps/51.1.81.

Kucharzewski, M., \& Kuczma, M. (1964). Basic concepts of the theory of geometric objects. Warsaw: Państwowe Wydawnictwo Naukowe.

Ladyman, J., \& Ross, D. (2007). Every thing must go: Metaphysics naturalised. Oxford: Oxford University Press.

Lam, V. (2011). Gravitational and nongravitational energy: The need for background structures. Philosophy of Science, 78(5), 1012-1024. https://doi.org/10.1086/662260.

Lam, V., \& Wüthrich, Chr. (2018). Spacetime is as spacetime does. Studies in History and Philosophy of Modern Physics. https://doi.org/10.1016/j.shpsb.2018.04.003.

Le Bihan, B. (forth.). Spacetime emergence in quantum gravity: Functionalism and the hard problem. Synthese. Preprint: http://philsci-archive.pitt.edu/16560/.

Lehmkuhl, D. (2008). Is spacetime a gravitational field? In D. Dieks (Ed.), The ontology of spacetime (p. 2008). Amsterdam: Elsevier.

Lehmkuhl, D. (2011). Mass-Energy-Momentum: Only there because of spacetime? The British Journal for the Philosophy of Science, 62(3), 453-488. https://doi.org/10.1093/bjps/axr003.

Lehmkuhl, D. (2014). Why Einstein did not believe that general relativity geometrizes gravity. Studies in History and Philosophy of Modern Physics, 46(Part B), 316-326. https://doi.org/10.1016/j.shpsb. 2013.08.002.

Lehmkuhl, D. (2017). Literal versus careful interpretations of scientific theories The vacuum approach to the problem of motion in general relativity. Philosophy of Science, 84(5), 1202-1214. https://doi.org/ $10.1086 / 694398$.

Levin, J. (2013). Functionalism. Stanford Encyclopedia of Philosophy. https://plato.stanford.edu/entries/ functionalism/. Accessed Nov 2019.

Lewis, D. (1970). How to define theoretical terms. The Journal of Philosophy, 67(13), 427-446.

Lewis, D. (1972). Psychophysical and theoretical identifications. Australian Journal of Philosophy, 50(3), 249-258.

Ludvigsen, M. (1999). General relativity-A geometric approach. Cambridge: Cambridge University Press.

Maggiore, M. (2007). Gravitational waves. Volume 1: Theory and experiment. Oxford: Oxford University Press.

Maudlin, T. (2011). Quantum non-locality and relativity: Metaphysical intimations of modern physics. Chichester: Blackwell-Wiley. 
Maudlin, T. (2012). Philosophy of physics: Space and time. Princeton: Princeton University Press.

Misner, Ch., Thorne, K., \& Wheeler, J. (1973). Gravitation. New York: W.H. Freeman.

Murgueitio Ramirez, S. (2019). A puzzle concerning local symmetries and their empirical significance. http://philsci-archive.pitt.edu/16509/. Accessed Nov 2019.

Murphy, G. L. (1990). Energy and momentum from the Palatini formalism. International Journal of Theoretical Physics, 29(9), 1990. https://doi.org/10.1007/BF00673686.

Nerlich, G. (1991). How Euclidean geometry has misled metaphysics. In G. Nerlich (Ed.), What spacetime explains. Metaphysical essays on space and time. Cambridge: Cambridge University Press.

Nerlich, G. (2007). What spacetime explains. Metaphysical essays on space and time. Cambridge: Cambridge University Press.

Nerlich, G. (2013). Einstein's genie. Spacetime out of the bottle. Montreal: Minkowski Institute Press.

Nester, J. (2004). General pseudotensors and quasilocal quantities. Classical and Quantum Gravity, 21(S261), 2004.

Ney, A. (2010). The status of our ordinary three dimensions in a quantum universe. Noûs, 46(3), 525-560. https://doi.org/10.1111/j.1468-0068.2010.00797.x.

Ney, A. (forth.). Finding the world in the wavefunction: some strategies for solving the macro-object problem. Synthese. https://doi.org/10.1007/s11229-017-1349-4.

Nijenhuis, A. (1952). Theory of the geometric object. PhD thesis, University of Amsterdam, NL.

Norton, J. (1985). What was Einstein's principle of equivalence? Studies in History and Philosophy of Science, 16(1985), 203-246. https://doi.org/10.1016/0039-3681(85)90002-0.

Norton, J. (1989). Coordinates and covariance: Einstein's view of spacetime and the modern view. Foundations of Physics, 19, 1215-1263. https://doi.org/10.1007/BF00731880.

Norton, J. (2002). Einstein's triumph over the spacetime coordinate system. http://philsci-archive.pitt.edu/ 381/. Accessed Nov 2019.

Norton, J. (2012). Approximation and idealization. Why the difference matters. Philosophy of Science, 79(2), 207-232. https://doi.org/10.1086/664746.

Novotný, J. (1993). Energy-momentum complex of gravitational field in the Palatini formalism. International Journal for Theoretical Physics, 32(6), 1993. https://doi.org/10.1007/BF01215309.

Pauli, W. (1918). Theory of relativity. New York: Dover, 1981 (reprint).

Petrov, A. (2008). Nonlinear perturbations and conservation laws on curved backgrounds in GR and other metric theories. In: M. Christiansen/T. Rasmussen (eds.). Classical and Quantum Gravity Research. pp. 79-160. Hauppage: Nova Science Publishers. Preprint: https://arxiv.org/abs/0705.0019.

Pitts, J. B. (2006). Absolute objects and counterexamples: Jones-Geroch dust, torretti constant curvature, tetrad-spinor, and scalar density. Studies in History and Philosophy of Modern Physics, 37(2006), 347-371. https://doi.org/10.1016/j.shpsb.2005.11.004.

Pitts, J. B. (2009). Gauge-invariant localization of infinitely many gravitational energies from all possible auxiliary structures. General Relativity and Gravitation, 42(3), 601-622. https://doi.org/10.1007/ s10714-009-0874-y.

Pitts, J. B. (2011a). Massive Nordström scalar (density) gravities from universal coupling. General Relativity and Gravitation, 43(871-895), 2011. https://doi.org/10.1007/s10714-010-1100-7.

Pitts, J. B. (2011b). Permanent underdetermination from approximate empirical equivalence in field theory: Massless and massive scalar gravity, neutrino, electromagnetic, Yang-Mills and gravitational theories. British Journal for the Philosophy of Science, 62(2), 259-299. https://doi.org/10.1093/bjps/axq014.

Pitts, J. B. (2012). The nontriviality of trivial general covariance: How electrons restrict 'time' coordinates, spinors (almost) fit into tensor calculus, and 7/16 of a tetrad is surplus structure. Studies in History and Philosophy of Modern Physics, 43(2012), 1-24. https://doi.org/10.1016/j.shpsb.2011.11.001.

Pitts, J. B. (2016a). Space-time philosophy reconstructed via massive Nordström scalar gravities? Laws vs. geometry, conventionality, and underdetermination. Studies in History and Philosophy of Modern Physics, 53, 73-92. https://doi.org/10.1016/j.shpsb.2015.10.003.

Pitts, J. B. (2016b). Einstein's physical strategy, energy conservation, symmetries, and stability: 'But Grossmann \& I believed that the conservation laws were not satisfied. Studies in History and Philosophy of Modern Physics, 54, 52-72. https://doi.org/10.1016/j.shpsb.2016.04.003.

Pitts, J. B. (2016c). Einstein's equations for spin 2 mass 0 from Noether's converse Hilbertian Assertion. Studies in History and Philosophy of Modern Physics, 56(2016), 60-69. https://doi.org/10.1016/j. shpsb.2016.08.008.

Pitts, J. B. (2017). Progress and gravity. In K. Khamkham, J. Silk, \& S. Saunders (Eds.), Philosophy of cosmology. Cambridge: Cambridge University Press. https://doi.org/10.1017/9781316535783.014. 
Pitts, J.B. (2019). Cosmological constant $\Lambda$ vs. massive gravitations: A case study in general relativity exceptionalism vs. particle physics egalitarianism. In A. Blum et al. (Eds.), Back with a flourish: Revisiting the renaissance of general relativity. Birkhäuser. Preprint: https://arxiv.org/abs/1906.02115.

Pitts, J. B., \& Schieve, W. (2007). Universally coupled massive gravity. Theoretical and Mathematical Physics, 151(700-717), 2007. https://doi.org/10.1007/s11232-007-0055-7.

Poisson, E. (2004). A relativist's toolkit: The mathematics of black hole mechanics. Cambridge: Cambridge University Press.

Poisson, E., \& Will, C. M. (2014). Gravity. Newtonian, post-newtonian, relativistic. Cambridge: Cambridge University Press.

Pooley, O. (2013). Substantivalist and relationalist approaches to spacetime. In R. Batterman (Ed.), The oxford handbook of philosophy of physics (pp. 522-586). Oxford: Oxford University Press.

Pooley, O. (2017). Background independence, diffeomorphism invariance, and the meaning of coordinates. In D. Lehmkuhl (Ed.), Towards a theory of spacetime theories (pp. 105-143). Boston: Birkhäuser.

Read, J. (2018). Functional gravitational energy. British Journal for the Philosophy of Science. https://doi. org/10.1093/bjps/axx048.

Reiris, M. (2014). Stationary solutions and asymptotic flatness I\&II. https://arxiv.org/pdf/1002.1172.pdf, https://arxiv.org/abs/1310.0339.

Rey, D. (2013). Similarity assessment: Spacetime and the gravitational field-What does the metric tensor represent in general relativity? http://philsci-archive.pitt.edu/9615/. Accessed Nov 2019.

Rubin, D., \& Hayden, B. (2016). Is the expansion of the universe accelerating? All signs point to yes! Astrophysical Journal Letters, 833, L30. https://doi.org/10.3847/2041-8213/833/2/L30.

Schouten, J. A. (1954). Ricci calculus: An introduction to tensor and its geometrical applications (p. 1954). Berlin: Springer.

Schroedinger, E. (1950). Space-time structure. Cambridge: Cambridge University Press.

Schutz, B. (2012). Thoughts about a conceptual framework for relativistic gravity. In C. Lehner, J. Renn, \& M. Schemmel (Eds.), Einstein and the changing worldviews of physics. Boston: Birkhäuser.

Schutz, B., \& Ricci, F. (2010). Gravitational waves, sources, and detectors. https://arxiv.org/abs/1005.4735. Straumann, N. (2013). General relativity. With applications to astrophysics. Heidelberg: Springer.

Strevens, M. (2011). Depth: An account of scientific explanation. Cambridge: Harvard University Press.

Sus, A. (2017). The physical significance of symmetries from the perspective of conservation laws. In D. Lehmkuhl, G. Schliemann, \& E. Scholz (Eds.), Towards a theory of spacetime theories. Boston: Birkhaeuser.

Synge, J. L. (1960). Relativity. The general theory. Amsterdam: North-Holland Publishing Company.

Szabados, L. (2009). Quasi-local energy-momentum and angular momentum in general relativity. Living Reviews in Relativity, 12, 4.

Teh, N. J. (2015). Galileos gauge: Understanding the empirical significance of gauge symmetry. Philosophy of Science, 83(1), 93-118.

Torretti, R. (1990). Creative understanding: philosophical reflections on physics. Chicago: Chicago University Press.

Torretti, R. (1996). Relativity and geometry. New York: Dover Publications.

Trautman, A. (1962). Conservation laws in general relativity. In L. Witten (Ed.), Gravitation: Introduction to current research. San Francisco: Wiley.

Trautman, A. (1965). Foundations and current problems of General Relativity. In S. Deser \& K. W. Ford (Eds.), Lectures on general relativity. Englewood Cliffs, New Jersey: Prentice Hall.

Van Fraassen, B. (1980). The scientific image. Oxford: Oxford University Press.

Van Gulick, R. (2009). Functionalism. In B. McLaughlin, A. Beckermann, \& S. Walter (Eds.), Oxford handbook to the philosophy of mind (p. 2009). Oxford: Oxford University Press.

Vickers, P. (2016). Towards a realistic success-to-truth inference for scientific realism. Synthese. https:// doi.org/10.1007/s11229-016-1150-9.

Vickers, P. (2017). Understanding the selective realist defence against the PMI. Synthese, 194, 3221. https:// doi.org/10.1007/s11229-016-1082-4.

Visser, M., Barceló, C., \& Liberato, S. (2011). Analogue gravity. Living Reviews in Relativity, 10, 12. https://doi.org/10.12942/lrr-2011-3.

Wald, R. (1984). General relativity. Chicago: Chicago University Press.

Wallace, D. (2012). The emergent multiverse. Quantum theory according to the everett interpretation. Oxford: Oxford University Press. 
Wallace, D. (2016). Who's afraid of coordinate systems? An essay on representation of spacetime structure. Studies in History and Philosophy of Science B: Studies in History and Philosophy of Modern Physics. https://doi.org/10.1016/j.shpsb.2017.07.002.

Wallace, D. (2017). The case for black hole thermodynamics. Part I: Phenomenological thermodynamics", https://arxiv.org/abs/1710.02724.

Wallace, D., \& Greaves, H. (2014). Empirical consequences of symmetries. British Journal for the Philosophy of Science, 65(1), 59-89. https://doi.org/10.1093/bjps/axt005.

Weyl, H. (1921). Raum, Zeit, Materie. Vorlesungen ueber Relativitaetstheorie. Heidelberg: Springer, 1993 (reprint).

Will, C. (2014). The confrontation between general relativity and experiment. Living Review in Relativity. https://doi.org/10.12942/lrr-2014-4.

Woodward, J. (2014). Scientific explanation. stanford encyclopedia of philosophy. https://plato.stanford. edu/entries/scientific-explanation/. Accessed Nov 2019.

Xulu, S.S. (2003). The gravitational energy-momentum problem in general relativity. $\mathrm{PhD}$ thesis, University of Zululand, https://arxiv.org/pdf/hep-th/0308070.pdf.

Publisher's Note Springer Nature remains neutral with regard to jurisdictional claims in published maps and institutional affiliations. 